\title{
Prognostic and Immunological Significance of CXCR2 in Ovarian Cancer: A Promising Target for Survival Outcome and Immunotherapeutic Response Assessment
}

\author{
Haizhou Ji $\mathbb{D}^{1}{ }^{1}$ Mi Ren, ${ }^{2}$ Tongyu Liu, ${ }^{1}$ and Yang Sun $\mathbb{D}^{1}$ \\ ${ }^{1}$ Department of Gynecologic Oncology, Fujian Medical University Cancer Hospital, Fujian Cancer Hospital, Fuzhou, \\ 350014 Fujian, China \\ ${ }^{2}$ Department of Oncological Nursing, Fujian Medical University Cancer Hospital, Fujian Cancer Hospital, Fuzhou, \\ 350014 Fujian, China
}

Correspondence should be addressed to Haizhou Ji; 30492018@qq.com and Yang Sun; garyhanyu@sina.com

Received 7 October 2021; Revised 1 November 2021; Accepted 3 November 2021; Published 19 November 2021

Academic Editor: Fu Wang

Copyright (c) 2021 Haizhou Ji et al. This is an open access article distributed under the Creative Commons Attribution License, which permits unrestricted use, distribution, and reproduction in any medium, provided the original work is properly cited.

Objective. Uncovering genetic and immunologic tumor features is critical to gain insights into the mechanisms of immunotherapeutic response. Herein, this study observed the functions of CXCR2 in prognosis and immunology of ovarian cancer. Methods. Expression, prognostic significance, and genetic mutations of CXCR2 were analyzed in diverse cancer types based on TCGA and GTEx datasets. Associations of CXCR2 expression with immune checkpoints, neoantigens, tumor mutational burden (TMB), and microsatellite instability (MSI) were evaluated across pancancer. CXCR2-relevant genes were identified, and their biological functions were investigated in ovarian cancer. Through three algorithms (TIMER, quanTIseq, and xCell), we assessed the relationships of CXCR2 with immune cell infiltration in ovarian cancer. GSEA was adopted for inferring KEGG and hallmark pathways involved in CXCR2. Results. CXCR2 presented abnormal expression in tumors than paired normal tissues across pancancer. Higher expression of CXCR2 was found in ovarian cancer. Moreover, its expression was in relation to overall survival and progression including ovarian cancer. Prominent associations of CXCR2 with immune checkpoints, neoantigens, TMB, and MSI were observed in human cancers. Somatic mutations of CXCR2 frequently occurred across pancancer. Amplification was the main mutational type of CXCR2 in ovarian cancer. CXCR2-relevant genes were markedly enriched in immunity activation and carcinogenic pathways in ovarian cancer. Moreover, it participated in modulating immune cell infiltration in the tumor microenvironment of ovarian cancer such as macrophage and immune response was prominently modulated by CXCR2. Conclusion. Collectively, CXCR2 acts as a promising prognostic and immunological biomarker as well as a novel immunotherapeutic target of ovarian cancer.

\section{Introduction}

Ovarian cancer represents the major cause of deaths of gynecological malignancies $[1,2]$. Epithelial ovarian cancer is the most common form [3]. The five-year survival rate is $<35 \%$ globally [3]. 70\% of affected patients have advanced-stage disease [4]. The present first-line standards of care include debulking surgery plus platinum-taxane maintenance chemotherapeutic strategy [5]. Following the first-line treatment, cancer may relapse among $60-70 \%$ of patients with first-rank debulking as well as $80-85 \%$ of patients with sub- optimal debulking [6]. The high mortality of ovarian cancer patients can be attributed to chemotherapy resistance, extensive intraperitoneal metastasis, and other factors [7]. Awful mortality may be attributed chemotherapeutic resistance, extensive intraperitoneal metastases, etc. [8]. Tumor microenvironment exerts a critical role in the progression and clinical outcomes of ovarian cancer [9]. Emerging immunotherapeutic strategies enhance the antitumor immune response by diverse methods such as immunostimulatory cytokine and tumor antigen vaccine as well as monoclonal antibody [10]. Though immunotherapy-relevant agents like 
olaparib may ameliorate ovarian cancer progression, there is no prominent breakthrough for its effective therapy [11]. The presence and absence of tumor-infiltrating lymphocytes are separately judged as "hot" tumor and "cold" tumor [12]. Therefore, because of high infiltration levels of tumorinfiltrating lymphocytes, "hot" tumor can respond to immunosuppressive checkpoint inhibitors. Nevertheless, despite the relatively increased tumor mutation burden (TMB) of ovarian cancer, it is still a "cold" tumor [13]. Thus, it is of importance to explore how to activate the immune system in "cold" tumor such as $\mathrm{T}$ cell and tumor-associated macrophage.

Chemokine receptor (CXCR) family (including CXCR17 ) is a type of G-protein-coupled receptors, abundant in 7 transmembrane motifs containing hydrophobic amino acids [14]. Among them, CXCR2 was originally thought to be a G protein-coupled transmembrane chemokine receptor expressed on neutrophil [15]. It possesses the functions in various leukocytes such as neutrophil, eosinophil, and monocyte as well as macrophage, which is related to immune response [16]. Targeting CXCR2 in myeloid-derived suppressor cells may improve antitumor immune response [17]. Emerging evidence suggests that CXCR2 is involved in the recruitment of immune cells as well as promotes angiogenesis, tumor growth, and metastases [18]. It exhibits high affinity for IL-8 and Gro-1 but low affinity for Gro-2, Gro-3, and epithelial neutrophil-activating peptide-78 [18]. Moreover, high expression of CXCR2 contributes to carcinogenesis in diverse cancer types, especially ovarian cancer [19]. Also, CXCR2-expressing ovarian cancer is aggressive with undesirable clinical outcomes [20]. CXCR2 is crucial for the acquisition of cisplatin chemoresistance of ovarian cancer cells [21]. Despite this, the functions of CXCR2 in prognosis and immunology in ovarian cancer remain ambiguous. In this study, we aimed to evaluate the prognostic and immunological significance of CXCR2 in ovarian cancer.

\section{Materials and Methods}

2.1. Data Acquisition. This study acquired the transcriptome data, follow-up information, and genetic mutation data of pancancer samples from The Cancer Genome Atlas (TCGA) project via Genomic Data Commons (GDC) web server [22]. Meanwhile, we curated transcriptome profiles of normal specimens from Genotype-Tissue Expression (GTEx) projects [23]. Gene Expression Profiling Interactive Analysis 2 (GEPIA2) web server (http://gepia2.cancer-pku.cn/) provides an accessible resource for gene expression analysis in tumor and normal specimens from TCGA and GTEx projects. CXCR2 expression was compared between tumor and normal specimens with the Wilcoxon test. "Survival" module of GEPIA was applied for assessing the correlations of CXCR2 expression with overall survival (OS) of diverse cancer types. CXCR2 expression was analyzed across distinct cancer pathological stages. Univariate cox regression analyses were presented for investigating the associations of CXCR2 expression with OS and disease-specific survival (DSS) for diverse cancer types in TCGA cohort.
2.2. Analysis of Associations between CXCR2 Expression and Immune Checkpoints, Neoantigens, TMB, and Microsatellite Instability (MSI). The known immune checkpoints were curated from previous research [24]. TMB was calculated as the number of somatic, coding, base substitutions, and insert-deletion alterations per megabase of the genome detected utilizing nonsynonymous and code-shifting indels with the detection limit of $5 \%$ [25]. The formula of TMB was as follows: $\mathrm{TMB}=\mathrm{Sn} \times 1,000,000 / n$, where $\mathrm{Sn}$ represented the absolute number of somatic alterations while $n$ represented the number of exon base coverage depth $\geq$ $100 \times$. The number of neoantigens [26] as well as MSI [27] was separately counted across pancancer. Through the Spearman correlation test, we assessed the associations of CXCR2 expression with immune checkpoints, neoantigens, TMB, and MSI in diverse cancer types.

2.3. Somatic Mutation Analysis. Somatic mutations were visualized across ovarian cancer specimens from TCGA dataset utilizing Maftools package [28]. Through cBio Cancer Genomics Portal (cBioPortal) platform (http://cbioportal .org/) [29], alteration frequency of CXCR2 was analyzed in diverse cancer types. Genomic mutations of CXCR2 contained copy number amplification, deep deletion, and missense mutation.

2.4. Differential Expression Analysis. In line with the median value of CXCR2 expression, ovarian cancer specimens in TCGA dataset were classified into high- and low-expression groups. Limma package (version 3.40.2) was adopted for differential expression analysis between two groups [30]. With $\mid \log 2$ fold change $\mid>1$ and false discovery rate $(\mathrm{FDR})<0.05$, CXCR2-relevant genes were identified in ovarian cancer.

2.5. Function Enrichment Analysis. Biological processes of Gene Ontology (GO) and Kyoto Encyclopedia of Genes and Genomes (KEGG) pathways enriched by CXCR2relevant genes were analyzed with clusterProfiler package [31]. Terms with FDR $<0.05$ were significantly enriched.

2.6. Analysis of Immune Cell Infiltration. Three algorithms including Tumor Immune Estimation Resource (TIMER; http://cistrome.shinyapps.io/timer) [32], quanTIseq (http:// icbi.at/quantiseq) [33], and xCell (http://xCell.ucsf.edu/) [34] were employed for inferring the infiltration levels of immune cells in ovarian cancer from TCGA dataset. The Spearman correlation test was utilized to evaluate the relationships of CXCR2 expression with immune cell infiltrations.

2.7. Gene Set Enrichment Analysis (GSEA). For exploring the biological signaling pathways involved in CXCR2, GSEA software (version 4.0.3) [35] was adopted carried out between high- and low-expression groups with the median value of CXCR2 expression as the cutoff value. The first three or four terms of KEGG and hallmark were visualized. The gene sets of KEGG and hallmark pathways were curated from the Molecular Signature Database (MsigDB; http://www.broadinstitute.org/msigdb) [36]. KEGG or hallmark pathways with |nominal enrichment score (NES) |> 


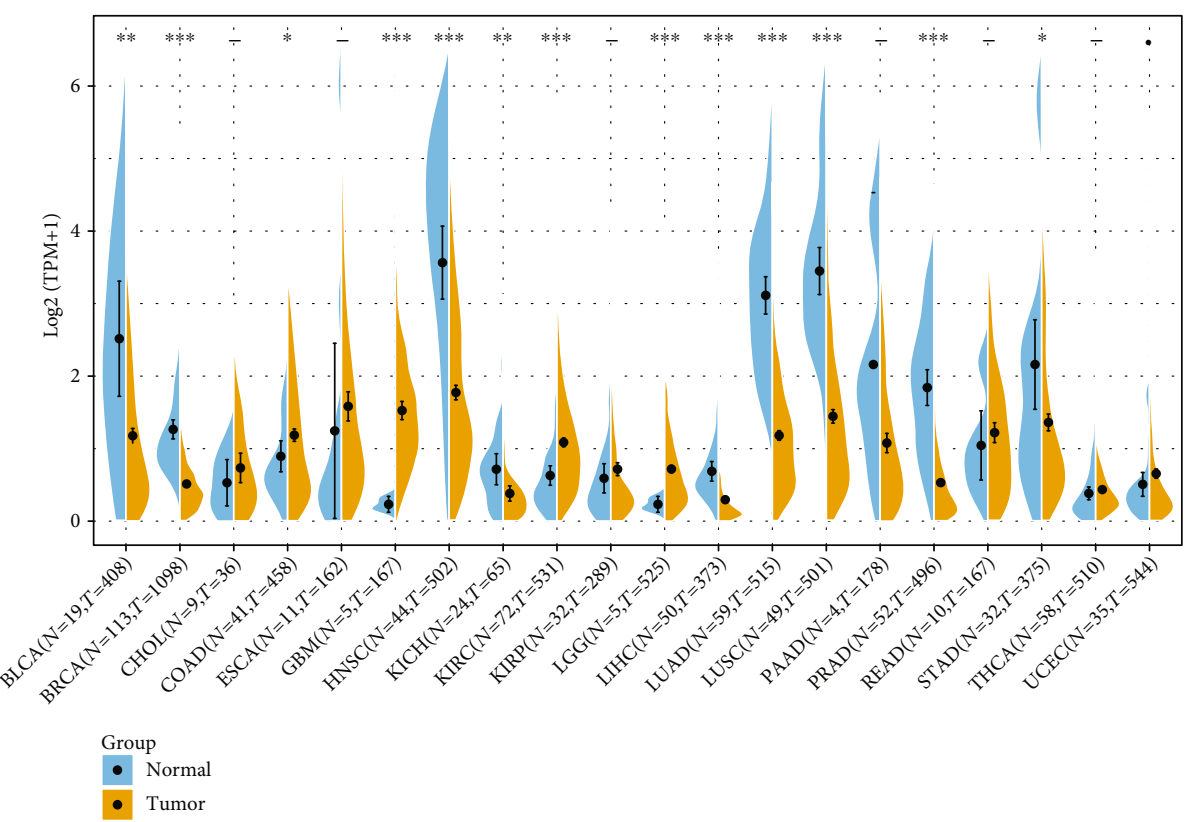

(a)

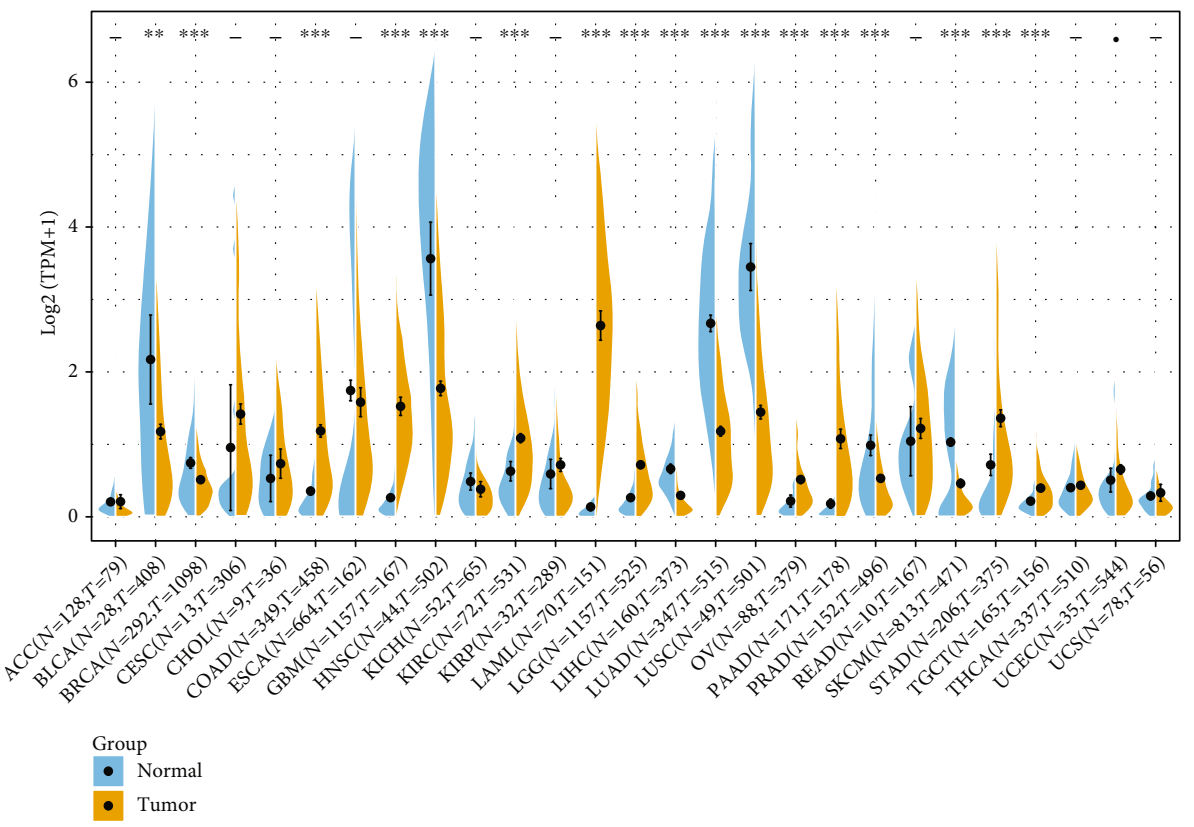

(b)

FIgURE 1: Continued. 

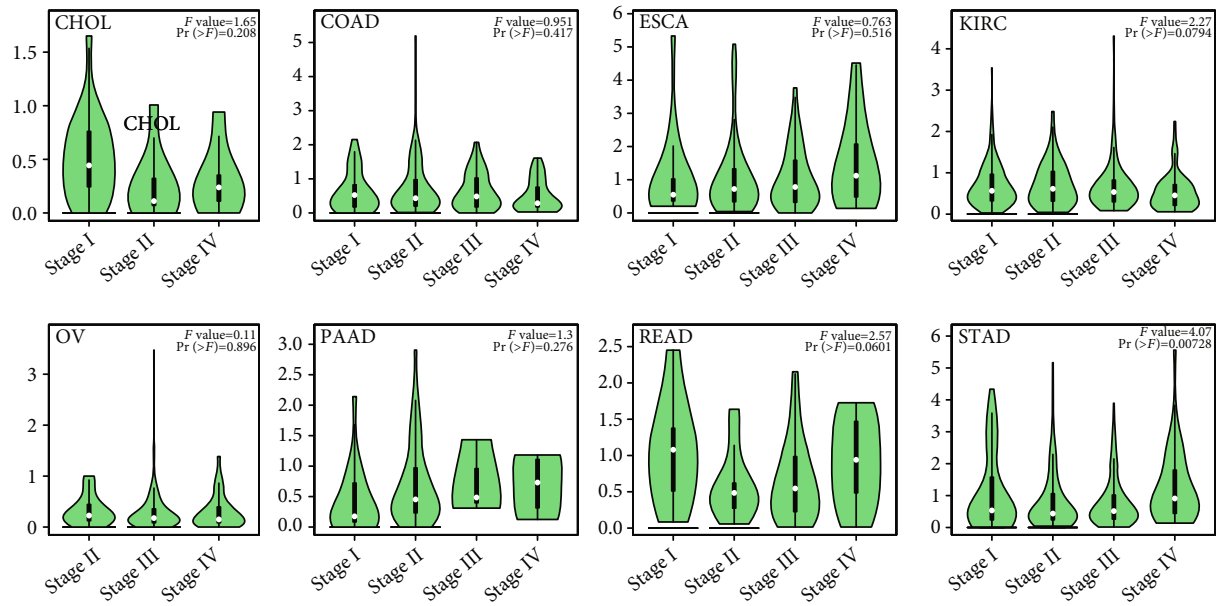

(c)

FIGURE 1: Expression patterns of CXCR2 across pancancer. (a) Expression levels of CXCR2 in tumor and normal tissues in TCGA dataset. (b) Expression levels of CXCR2 in tumor and normal tissues in TCGA and GTEx datasets. Yellow fusiformis represented tumor samples while blue fusiformis represented normal samples. The $X$-axis meant number of tumor and normal specimens. The $Y$-axis meant log 2( transcript per million $(\mathrm{TPM})+1) .{ }^{*} p<0.05 ;{ }^{*} p<0.01 ;{ }^{* * *} p<0.001$. (c) Expression levels of CXCR2 in different pathological stages across pancancer.

1.7 and nominal $p<0.05$ were considered to have significant enrichment.

2.8. Statistical Analysis. All statistics were presented with $\mathrm{R}$ software (version 4.0.3; https://www.R-project.org) and its packages. Comparisons between groups were conducted with Student's $t$-test, the Wilcoxon test, or one-way variance analyses. The Spearman or Pearson correlation test was utilized for evaluating correlations between variables. A $p$ value $<0.05$ was set as statistically significant.

\section{Results}

3.1. Expression Patterns of CXCR2 across Pancancer. Herein, this study evaluated the expression of CXCR2 in diverse tumor tissues and matched normal tissues. We collected data from TCGA and GTEx datasets. In TCGA dataset, we noticed high expression of CXCR2 in COAD, GBM, KIRC, and LGG (Figure 1(a)). In contrast, CXCR2 displayed reduced expression in BLCA, BRCA, HNSC, KICH, LIHC, LUAD, PAAD, PRAD, and STAD. Due to the relatively small sample size of normal tissues in TCGA, we integrated data from TCGA and GTEx datasets. There was reduced expression of CXCR2 in BLCA, BRCA, COAD, GBM, HNSC, LIHC, LUAD, LUSC, PRAD, and SKCM (Figure 1(b)). Nevertheless, upregulation of CXCR2 expression was found in KIRC, LAML, LGG, OV, PAAD, STAD, and TGCT. Using the GEPIA2 tool, the relationships of CXCR2 expression with pathological staging were evaluated in CHOL, COAD, ESCA, KIRC, OV, PAAD, READ, and STAD (Figure 1(c)). Among them, CXCR2 displayed stage-specific expression alterations in STAD, while no clear associations were found in most cancer types.
3.2. Prognostic Significance of CXCR2 across Pancancer. Through the GEPIA2 tool, we investigated the associations of CXCR2 with OS across diverse cancer types. The results demonstrated that CXCR2 upregulation was in relation to worse OS of OV and LGG patients (Figures 2(a)-2(c)). Oppositely, KIRC patients with high expression of CXCR2 displayed marked survival advantage in comparison to those with low expression of CXCR2 (Figure 2(d)). Furthermore, univariate cox regression models were conducted for investigating the correlations of CXCR2 with OS and DSS in each cancer type. In Figure 2(e), we noticed CXCR2 as a risk factor for OS of ACC, DLBC, LAML, LGG, OV, and STAD. In contrast, CXCR2 acted as a protective factor of MESO OS. Moreover, CXCR2 upregulation displayed worse DSS for ACC, DLBC, LGG, OV, and STAD (Figure 2(f)). Kaplan-Meier curves also demonstrated the prognostic significance of CXCR2 in OS and DSS of diverse cancer types (Supplementary figure 1A, B).

\subsection{Analysis of Links between CXCR2 Expression and Tumor} Immune Response across Pancancer. Nowadays, several genes have been recognized as immune checkpoints in tumor immune response. We evaluated whether there is a link of CXCR2 with immune checkpoint genes. The results demonstrated markedly positive associations between CXCR2 and immune checkpoint genes such as CD86, VSIR, CD28, and CTLA4 across pancancer (Figure 3(a)). For uncovering the function of CXCR2 in the immune mechanism and immune response, this study evaluated the interactions of CXCR2 expression with neoantigens, TMB, and MSI. Neoantigens, $\mathrm{TMB}$, and MSI are in relation to antitumor immunity and may predict therapeutic responses to immunotherapeutic agents. Correlation between CXCR2 expression and neoantigens was assessed in diverse cancer types. In Figure 3(b), CXCR2 exhibited prominently negative associations with 


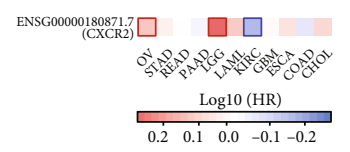

(a)

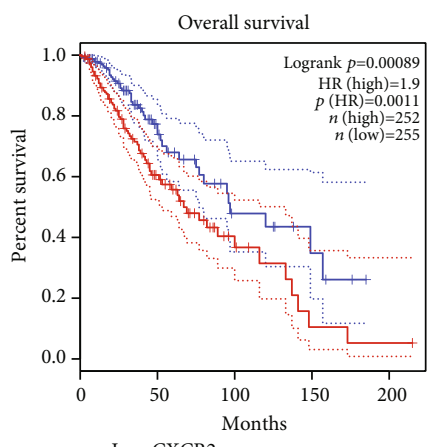

— Low CXCR2 group
- High CXCR2 group

(c)

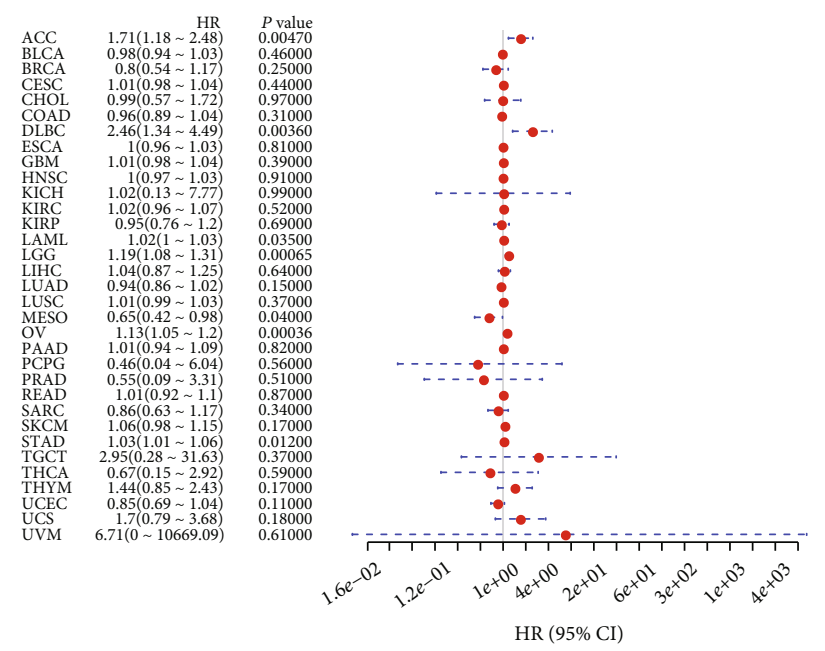

(e)

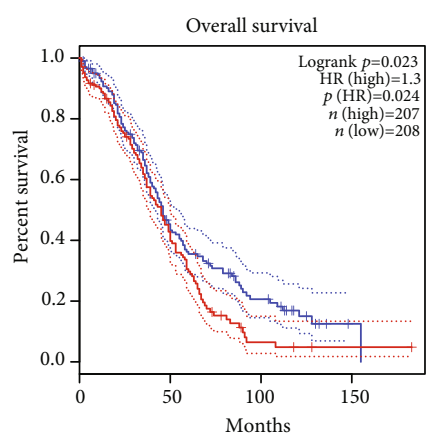

— Low CXCR2 group

(b)

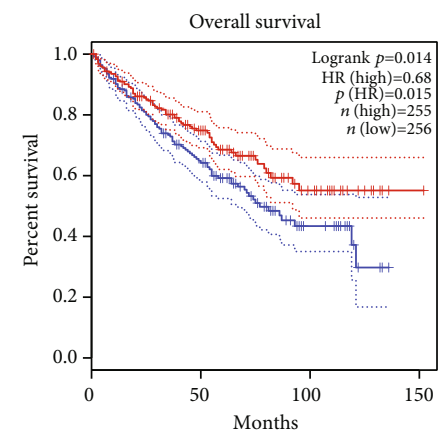

— Low CXCR2 group
— High CXCR2 group

(d)
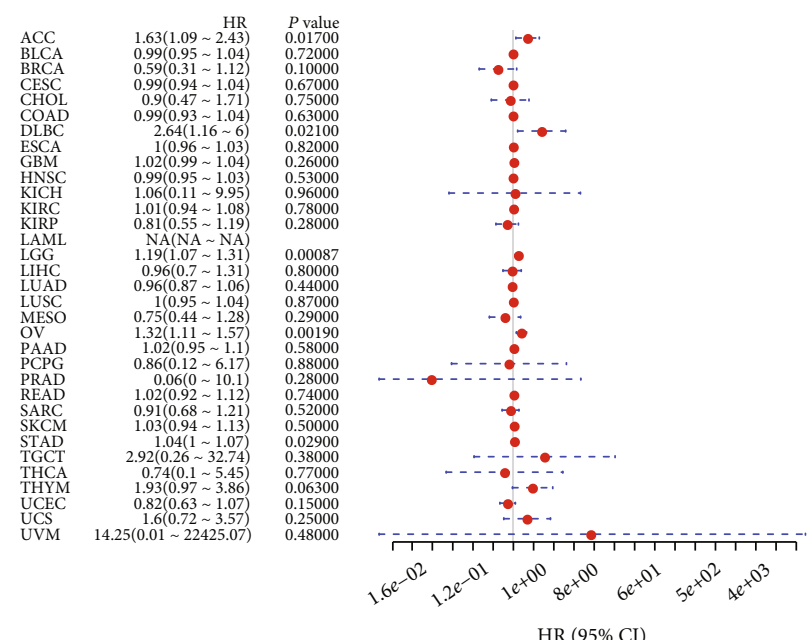

(f)

FIGURE 2: Evaluation of prognostic significance of CXCR2 across pancancer. (a) Survival map for the associations of CXCR2 with OS of diverse cancer types. Red meant HR $>1$ while blue meant $\mathrm{HR}<1$. (b-d) Kaplan-Meier curves of high and low expression of CXCR2 groups for OV, LGG, and KIRC patients. (e, f) Univariate cox regression analysis showing the associations of CXCR2 with OS and DSS across diverse cancer types.

the number of neoantigens in BRCA, SKCM, BLCA, and PRAD. Moreover, we noticed the negative links of CXCR2 expression with TMB in BLCA, BRCA, LIHC, LUAD, PAAD, PRAD, and THCA (Figure 3(c)). However, CXCR2 expression displayed positive correlations to TMB in LGG and OV. As depicted in Figure 3(d), there were negative interactions between CXCR2 expression and MSI in CHOL,
ESCA, HNSC, KIRP, LGG, LUAD, LUSC, PAAD, PRAD, SKCM, STAD, UCEC, and UCS. The above evidence highlighted the implications of CXCR2 expression in tumor immune response.

3.4. Analysis of Somatic Mutation of CXCR2 in Ovarian Cancer. We analyzed the somatic mutation of CXCR2 in 


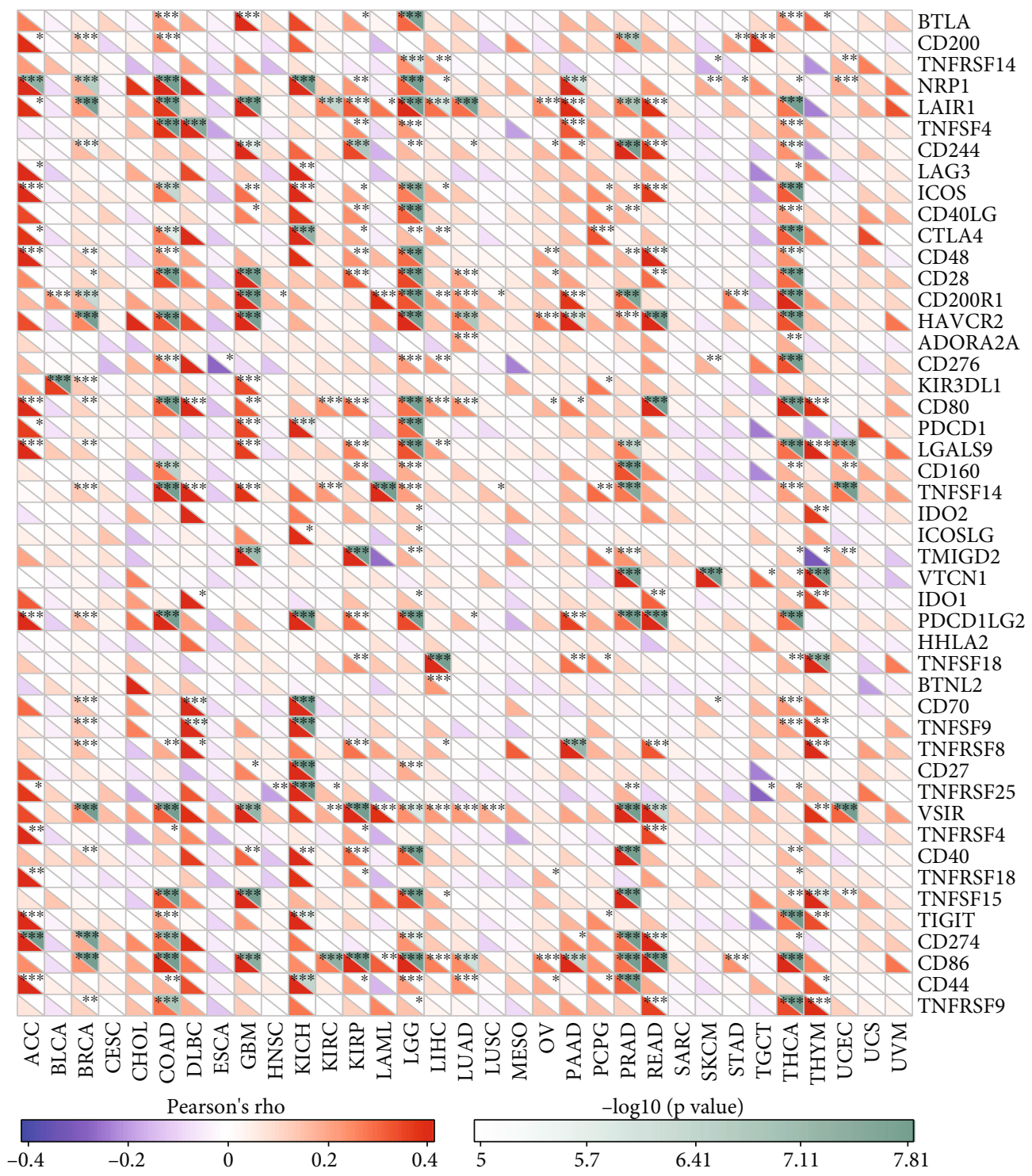

(a)

FIgure 3: Continued. 

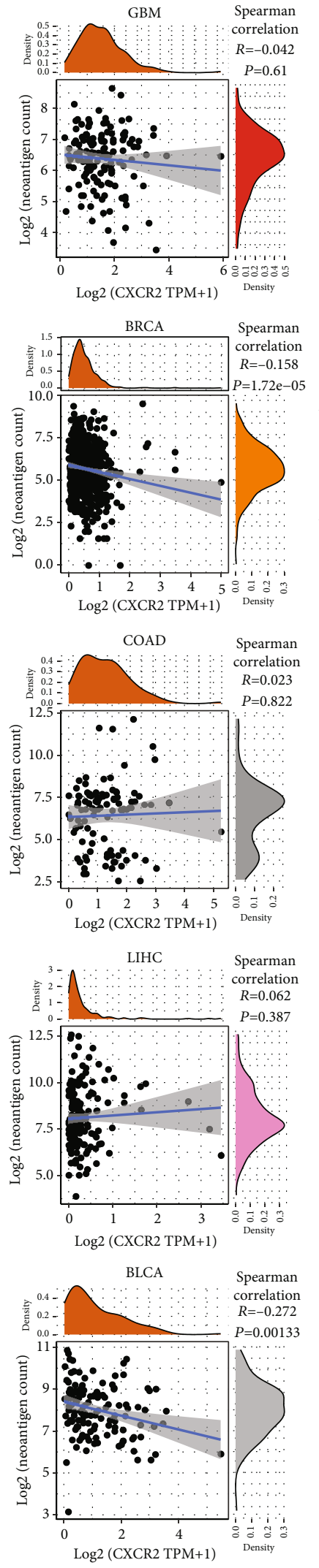
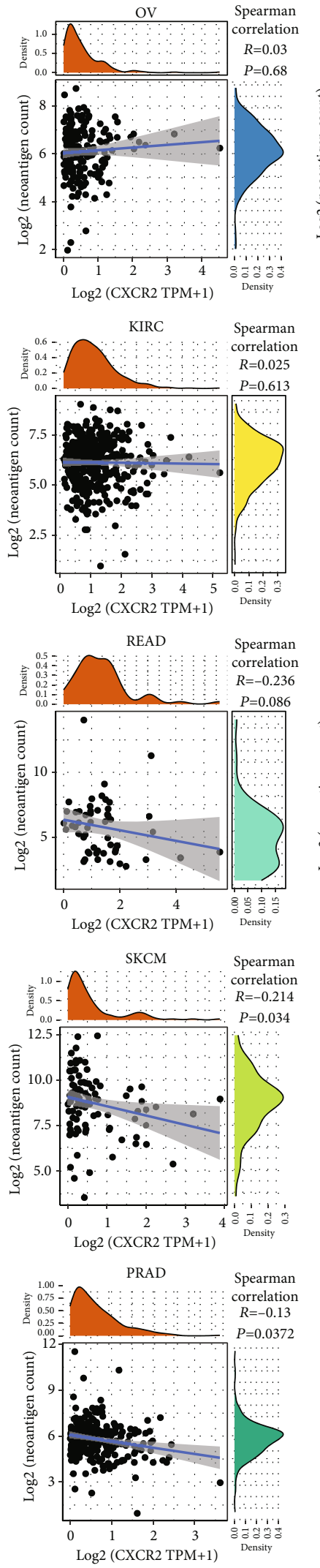
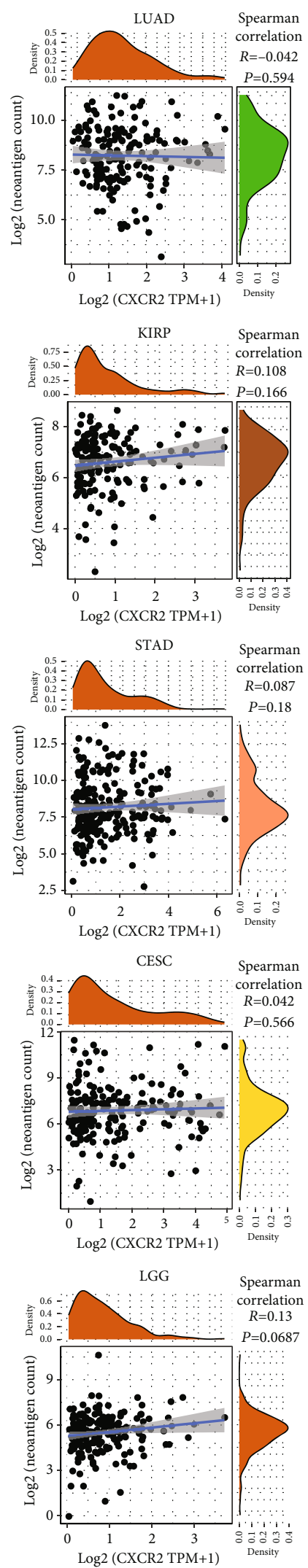

(b)
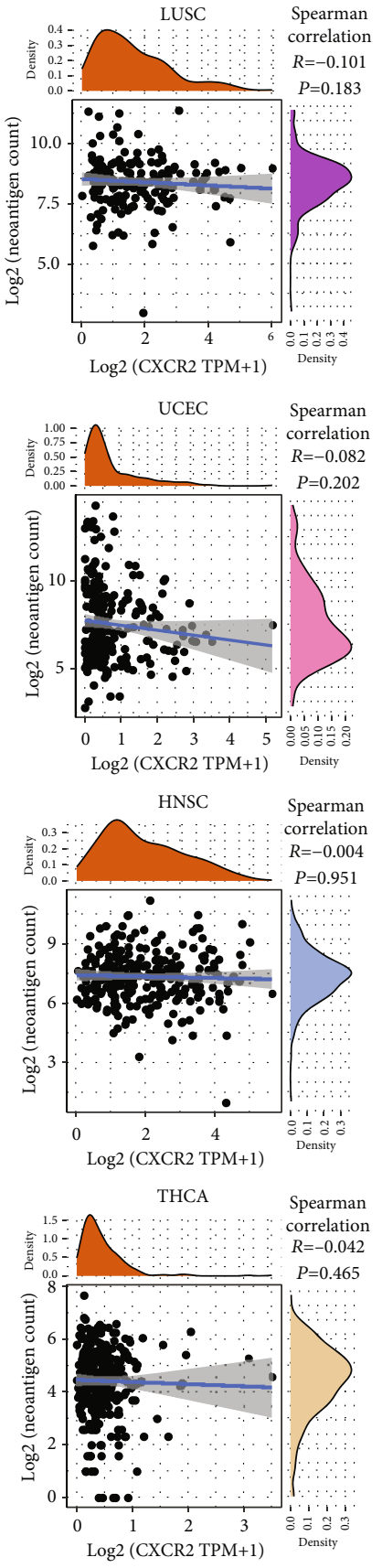

Figure 3: Continued. 


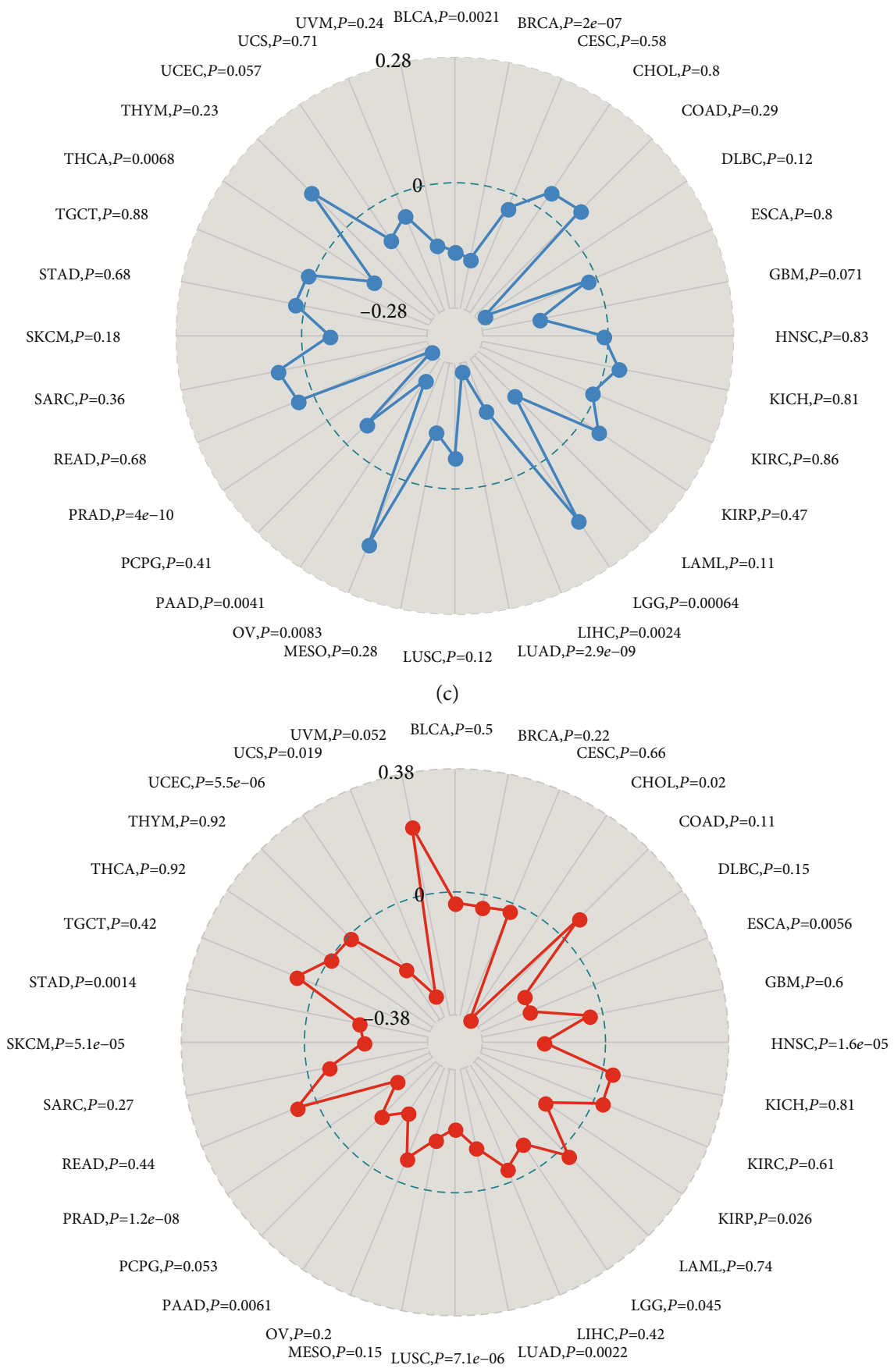

(d)

FIgURE 3: Associations of CXCR2 expression with tumor immune response across pan-cancer. (a) Correlations of CXCR2 expression with acknowledged immune checkpoint genes in diverse cancer types. The lower triangle meant coefficients calculated with Pearson's correlation test, while the upper triangle meant $p$ value. ${ }^{*} p<0.05,{ }^{* *} p<0.01$, and ${ }^{* * *} p<0.001$. (b) Correlation analysis between CXCR2 expression and the number of immune neoantigens across pancancer. (c) Association analysis of CXCR2 expression with TMB across pancancer utilizing Spearman's correlation test. (d) Association analysis of CXCR2 expression with MSI in diverse cancer types with Spearman's correlation test.

ovarian cancer. As shown in Figure 4(a), the somatic mutation rate was $0.46 \%$. Among 436 ovarian cancer samples, genetic mutations occurred in 261 (59.86\%) (Figure 4(b)). TP53 (56\%), TTN (23\%), CSMD3 (8\%), MUC16 (7\%), FLG (6\%), FAT3 (6\%), DNAH3 (5\%), SYNE1 (5\%), USH2A $(5 \%)$, and HMCN1 (4\%) were the most frequently mutated genes across ovarian cancer. Moreover, missense mutation was the major mutation type. However, no significant difference in genetic mutation was investigated between high and low expression of CXCR2 groups. Through cBioPortal tool, we evaluated the genetic mutation of CXCR2 across pancancer. We noticed that amplification of CXCR2 occupied the 
CXCR2 : [Somatic mutation rate: $0.46 \%$ ]

$\left.{ }_{1}^{5}\right]$

NM_001557

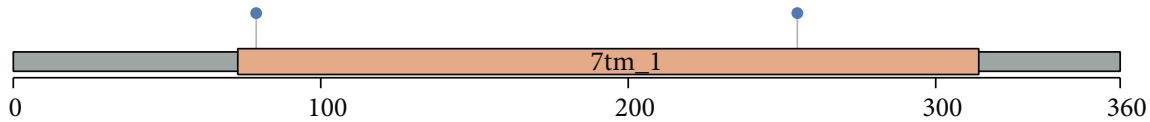

- Missense_Mutation

(a)

Altered in 261 (59.86\%) of 436 samples.

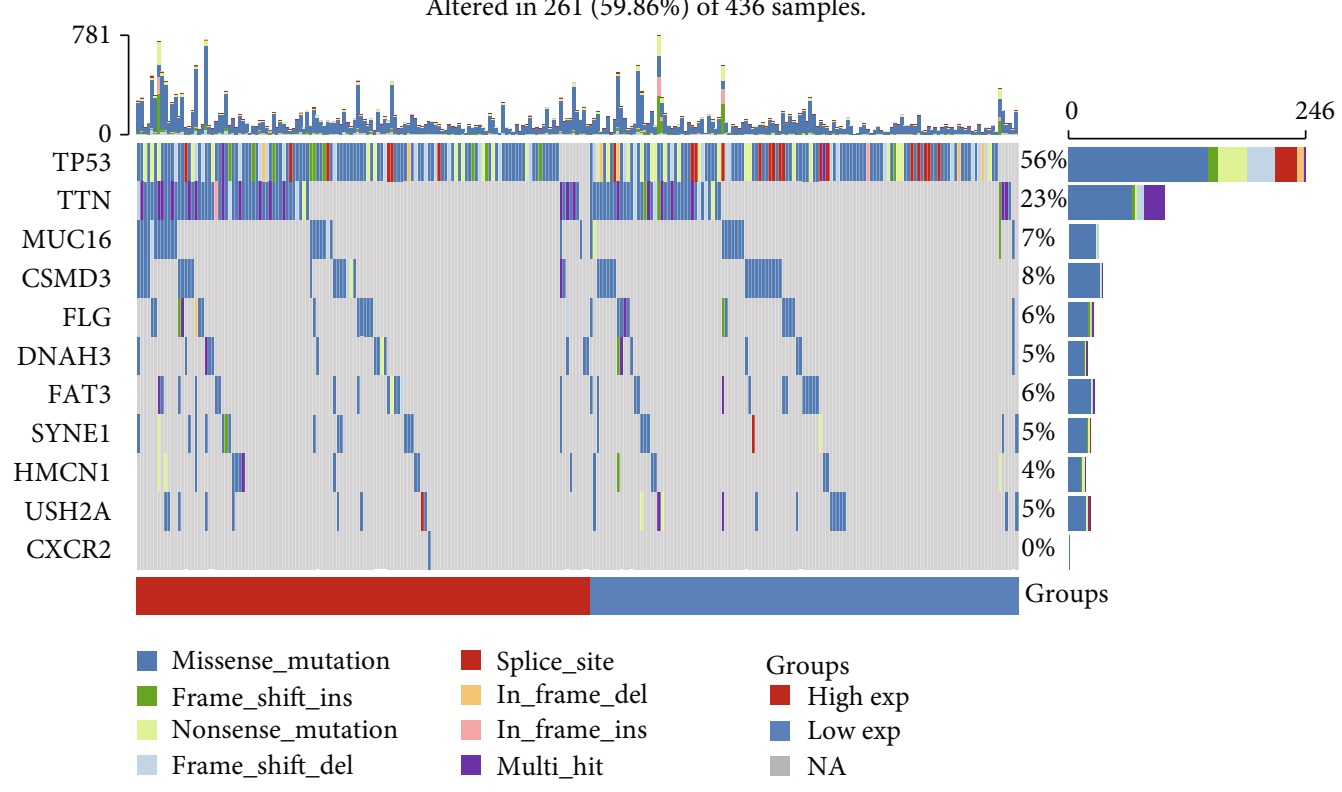

(b)

Figure 4: Continued. 


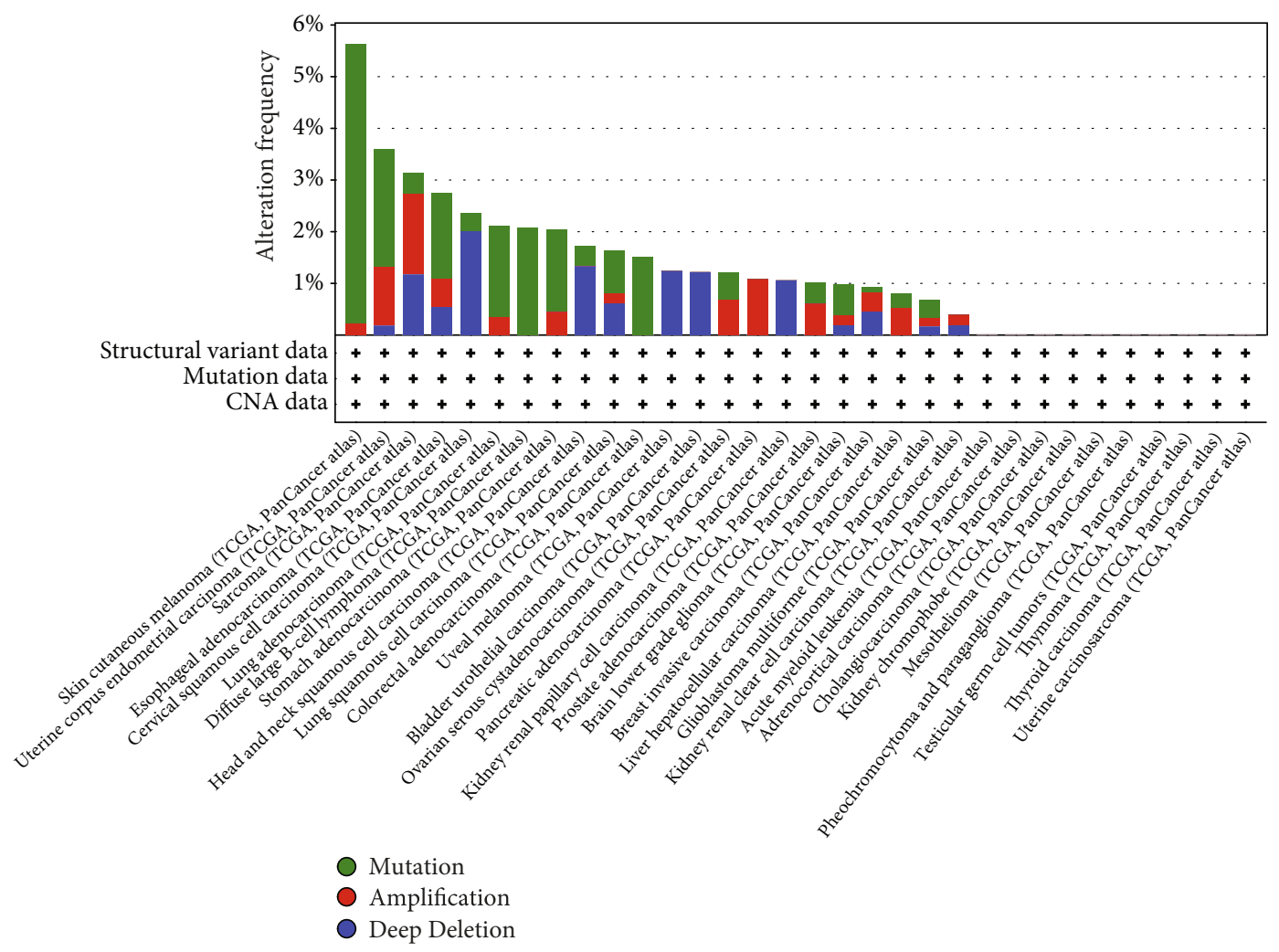

(c)

FIgURE 4: Analysis of somatic mutation of CXCR2 in ovarian cancer. (a) Somatic mutation rate of CXCR2 across ovarian cancer. (b) Landscape of genetic mutations across ovarian cancer specimens. Waterfall plots showed the mutational information of each gene in each specimen. Diverse colors at the bottom represented diverse mutational types. The barplot above the legend displayed the number of mutational burden. (c) Genetic mutation type and frequency of CXCR2 across pancancer via the cBioPortal tool. Histogram showed the alteration frequencies of CXCR2 in diverse cancer types. Green, mutation; red, amplification; and blue, deletion.

relatively high alteration frequency in ovarian cancer, which could contribute to the upregulation of CXCR2 expression (Figure 4(c)).

\subsection{Identifying CXCR2-Relevant Genes and Their Biological} Significance. To identify CXCR2-relevant genes, we separated ovarian cancer patients into high- and low-expression groups in line with the median value of CXCR2 expression. With $\mid \log 2$ fold change $\mid>1$ and $\mathrm{FDR}<0.05$, we screened 734 CXCR2-relevant genes (Figures 5(a) and 5(b)). Among them, 715 genes were upregulated while 19 genes were downregulated in the high-expression group (Tables 1 and 2). Function enrichment analysis was presented for uncovering the biological significance of CXCR2-relevant genes. In Table 3 and Figure 5(c), upregulated genes were in relation to KEGG pathways of immunity and inflammatory response such as Th17 cell differentiation, cytokine-cytokine receptor interaction, chemokine signaling pathway, antigen processing and presentation, human $\mathrm{T}$ cell leukemia virus 1 infection, graft-versus-host disease, and allograft rejection. Meanwhile, upregulated genes were prominently enriched by immune response like regulation of mononuclear, lymphocyte, and leukocyte proliferation; leukocyte cell-cell adhesion; $\mathrm{T}$ cell activation; myeloid leukocyte migration; neutrophil degranulation; and neutrophil activation involved in immune response (Table 4 and Figure 5(c)). Intriguingly, downregulated genes displayed significant correlations to carcinogenic pathways such as PPAR signaling pathway, neuroactive ligand-receptor interaction, melanoma, gastric cancer, cell adhesion molecules, and breast cancer (Table 5 and Figure 5(c)). Also, we noticed that downregulated genes were markedly associated with metabolic processes like triglyceride metabolic and catabolic processes, neutral lipid metabolic and catabolic processes, glycerolipid catabolic process, and acylglycerol metabolic and catabolic processes (Table 6 and Figure 5(c)).

3.6. Associations of CXCR2 with Immune Cell Infiltration in Tumor Microenvironment. Three algorithms (TIMER, quanTIseq, and xCell) were adopted for inferring the infiltration levels of immune cells in ovarian cancer. In Figure 6(a), correlation analysis uncovered that CXCR2 was negatively associated with the abundance of CD4+ T cell, neutrophil, myeloid dendritic cell, and macrophage in ovarian cancer tissues with TIMER algorithm. Using a quanTIseq method, we noticed the negative associations of CXCR2 with T regulatory cell (Treg), M1 macrophage, and M2 macrophage (Figure 6(b)). Oppositely, there were positive correlations 


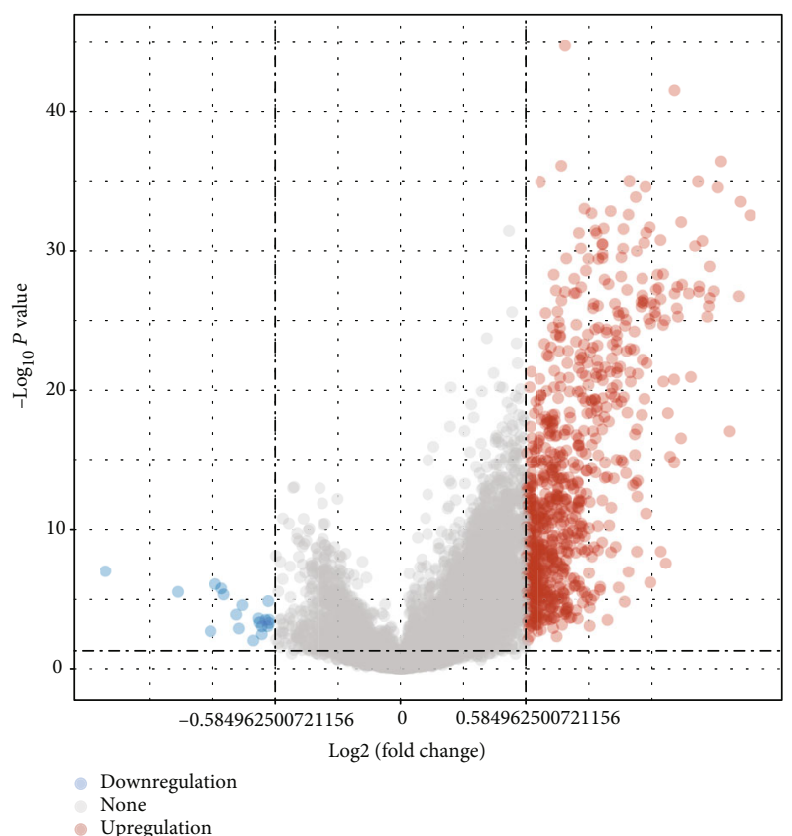

(a)

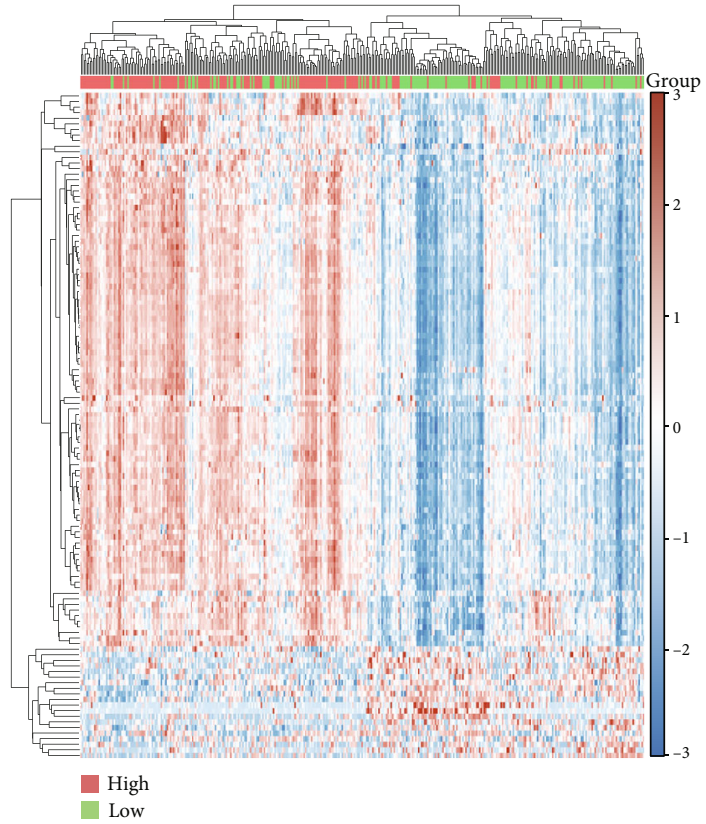

(b)

Figure 5: Continued. 


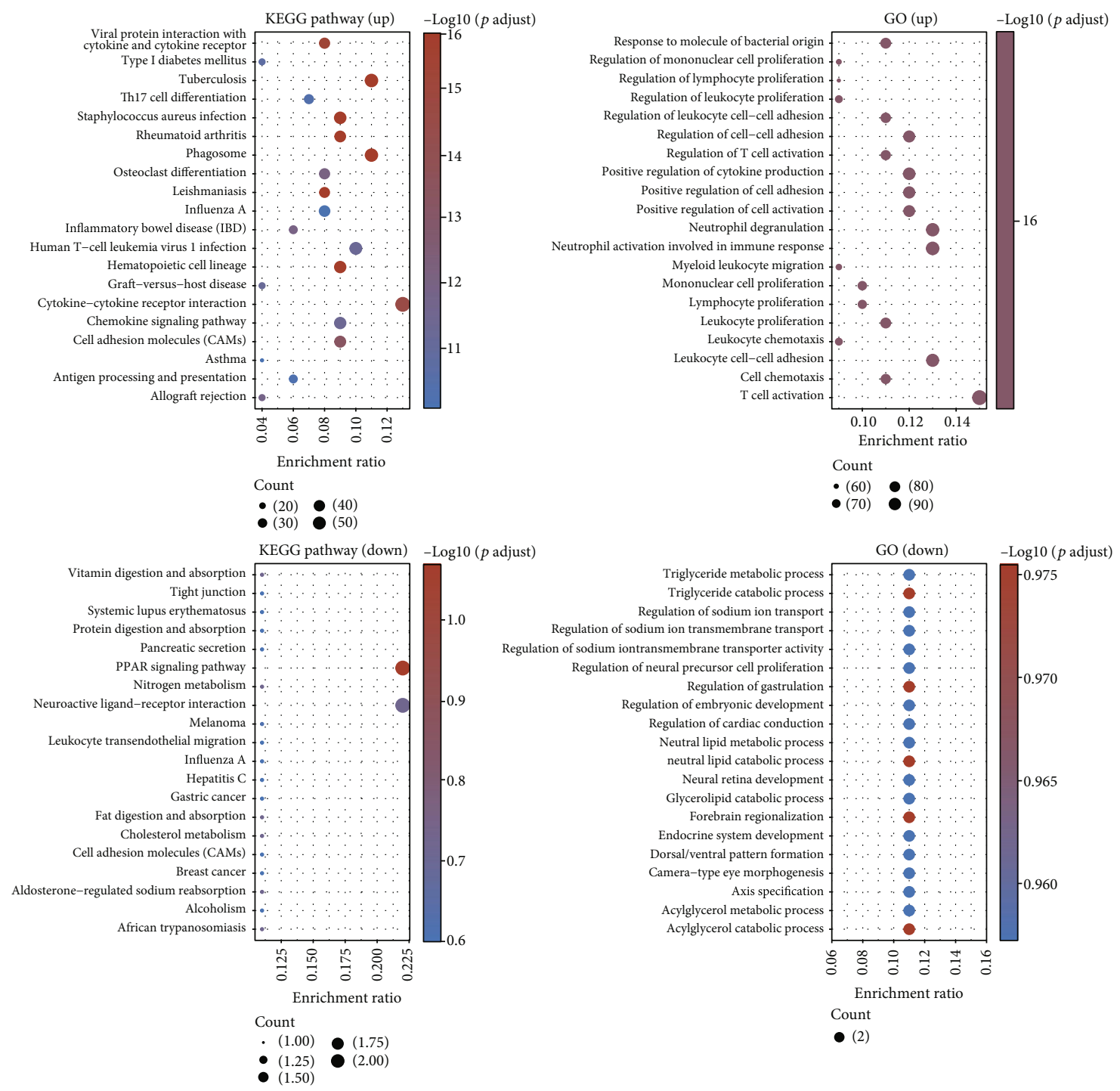

(c)

FIGURE 5: Identifying CXCR2-relevant genes in ovarian cancer and their biological significance. (a, b) Volcano plots and heat map visualized the expression patterns of CXCR2-relevant genes in high and low expression of CXCR2 groups. Red meant upregulation; blue meant downregulation; and grey meant no significant difference. (c) KEGG pathways and biological processes enriched by upregulated CXCR2relevant genes or downregulated CXCR2-relevant genes.

of CXCR2 with uncharacterized cell and CD4+ T cell. Through $x$ Cell algorithm, we investigated that CXCR2 displayed negative associations with stromal score, microenvironment score, immune score, CD8+ effector memory $\mathrm{T}$ cell, CD4+ naïve T cell, CD4+ effector memory T cell, neutrophil, activated myeloid dendritic cell, myeloid dendritic cell, monocyte, M1 macrophage, M2 macrophage, macrophage, hematopoietic stem cell, granulocyte-monocyte progenitor, endothelial cell, and common myeloid progenitor (Figure 6(c)). In contrast, we noticed the positive associations of CXCR2 with the abundance of CD8+ naïve T cell, CD4+ central memory T cell, CD4+ Th2 T cell, CD4+ Th1 $\mathrm{T}$ cell, common lymphoid progenitor, and $\mathrm{B}$ cell plasma across ovarian cancer. Based on three algorithms, CXCR2 expression negatively modulated macrophage infiltration in ovarian cancer.

3.7. Analysis of Signaling Pathways Involved in CXCR2. For observing the function of CXCR2 expression on tumor progression, this study separated ovarian cancer specimens into high- and low-expression groups in line with CXCR2 expression. Afterwards, we evaluated the enrichment of KEGG and hallmark pathways in high- and low-expression groups via GSEA. Our data suggested that CXCR2 exhibited negative correlations to leishmania infection, chemokine signaling pathway, and cytokine-cytokine receptor interaction KEGG pathways (Figure 7(a)). Meanwhile, there were positive relationships of CXCR2 with homologous recombination, 
TABLE 1: The first 20 upregulated CXCR2-relevant genes ranked by |log2fold change|.

\begin{tabular}{|c|c|c|c|c|c|c|}
\hline Genes & log2fold change & Average expression & $t$ & $p$ value & FDR & $B$ \\
\hline CYBB & 1.626883 & 4.866193 & 13.2859 & $2.82 E-33$ & $3.13 E-30$ & 64.80304 \\
\hline CSF1R & 1.580807 & 5.08034 & 13.53358 & $2.96 E-34$ & $4.76 E-31$ & 67.02763 \\
\hline ALOX5AP & 1.572744 & 5.250381 & 11.77966 & $1.82 E-27$ & $4.82 E-25$ & 51.62072 \\
\hline LYZ & 1.530011 & 6.245003 & 9.038626 & $8.60 E-18$ & $6.04 E-16$ & 29.71133 \\
\hline SLCO2B1 & 1.489949 & 3.454025 & 14.24883 & $4.06 E-37$ & $2.39 E-33$ & 73.52581 \\
\hline MPEG1 & 1.475656 & 3.816864 & 13.79154 & $2.78 E-35$ & $5.46 E-32$ & 69.35911 \\
\hline VSIG4 & 1.457472 & 5.213007 & 11.87514 & $7.96 E-28$ & $2.39 E-25$ & 52.43645 \\
\hline FPR3 & 1.439832 & 4.19429 & 11.74373 & $2.49 E-27$ & $6.48 E-25$ & 51.31448 \\
\hline LAPTM5 & 1.439653 & 7.33844 & 12.34081 & $1.35 E-29$ & $5.82 E-27$ & 56.45608 \\
\hline FCGR3A & 1.435916 & 5.415789 & 11.59154 & $9.24 E-27$ & $2.07 E-24$ & 50.02226 \\
\hline CX3CR1 & 1.428444 & 3.051211 & 11.38818 & $5.27 E-26$ & $1.01 E-23$ & 48.30796 \\
\hline C3AR1 & 1.406321 & 4.279677 & 12.81774 & $1.93 E-31$ & $1.27 E-28$ & 60.6387 \\
\hline ITGB2 & 1.391398 & 4.898533 & 11.96583 & $3.62 E-28$ & $1.23 E-25$ & 53.21399 \\
\hline CD163 & 1.390154 & 4.016596 & 11.86577 & $8.64 E-28$ & $2.55 E-25$ & 52.35628 \\
\hline GPR34 & 1.38616 & 3.462056 & 13.89462 & $1.08 E-35$ & $2.91 E-32$ & 70.29467 \\
\hline FPR1 & 1.372222 & 2.86155 & 12.72349 & $4.49 E-31$ & $2.57 E-28$ & 59.80717 \\
\hline $\mathrm{F} 13 \mathrm{~A} 1$ & 1.352817 & 3.950676 & 10.18952 & $1.10 E-21$ & $1.22 E-19$ & 38.51989 \\
\hline SIGLEC1 & 1.343363 & 3.306899 & 11.83175 & $1.16 E-27$ & $3.17 E-25$ & 52.06539 \\
\hline FGL2 & 1.311918 & 3.734005 & 11.99986 & $2.69 E-28$ & $9.33 E-26$ & 53.50643 \\
\hline MNDA & 1.30756 & 3.392344 & 13.16289 & $8.62 E-33$ & $8.98 E-30$ & 63.70358 \\
\hline
\end{tabular}

TABLE 2: The downregulated CXCR2-relevant genes ranked by |log2fold change|.

\begin{tabular}{|c|c|c|c|c|c|c|}
\hline Genes & log2fold change & Average expression & $t$ & $p$ value & FDR & $B$ \\
\hline PCP4 & -1.372 & 4.022248 & -5.43074 & $1.01 E-07$ & $1.15 E-06$ & 7.096412 \\
\hline APOA1 & -1.03739 & 6.955593 & -4.73466 & $3.12 E-06$ & $2.29 E-05$ & 3.80636 \\
\hline CLDN6 & -0.88582 & 6.125029 & -3.11872 & 0.001957 & 0.006165 & -2.2451 \\
\hline FXYD4 & -0.86449 & 1.906169 & -5.00148 & $8.76 E-07$ & $7.46 E-06$ & 5.021079 \\
\hline FGF17 & -0.83534 & 1.148663 & -4.85379 & $1.78 E-06$ & $1.40 E-05$ & 4.341469 \\
\hline SMIM24 & -0.82416 & 2.673542 & -4.64089 & $4.80 E-06$ & $3.34 E-05$ & 3.393485 \\
\hline SIX3 & -0.76623 & 1.360529 & -3.86787 & 0.000129 & 0.000589 & 0.274769 \\
\hline MAL & -0.75402 & 6.521845 & -3.25231 & 0.001249 & 0.004188 & -1.833 \\
\hline FXYD7 & -0.73799 & 2.355221 & -4.24556 & $2.75 E-05$ & 0.000153 & 1.734303 \\
\hline LHX1 & -0.68835 & 2.827502 & -2.62669 & 0.008976 & 0.02269 & -3.62052 \\
\hline PDCL2 & -0.66398 & 1.539734 & -3.70687 & 0.000241 & 0.001012 & -0.3093 \\
\hline NUPR2 & -0.65946 & 4.707309 & -3.53281 & 0.000462 & 0.001776 & -0.91478 \\
\hline SAMD11 & -0.64936 & 3.063171 & -3.35658 & 0.00087 & 0.003063 & -1.5 \\
\hline FABP6 & -0.64904 & 2.858451 & -2.96587 & 0.003212 & 0.009419 & -2.69647 \\
\hline NPW & -0.63225 & 5.019634 & -3.64286 & 0.000308 & 0.001243 & -0.53513 \\
\hline CA9 & -0.62153 & 4.176407 & -3.36072 & 0.000857 & 0.003025 & -1.48657 \\
\hline $\mathrm{H} 3 \mathrm{C} 11$ & -0.6192 & 2.105437 & -4.39824 & $1.42 E-05$ & $8.64 E-05$ & 2.359317 \\
\hline PRSS1 & -0.61512 & 3.467221 & -3.50754 & 0.000507 & 0.001923 & -1.00041 \\
\hline DEFB126 & -0.61494 & 1.807047 & -3.64746 & 0.000302 & 0.001224 & -0.51901 \\
\hline
\end{tabular}

base excision repair, proteasome, and DNA replication (Figure 7(b)). As depicted in Figure 7(c), hallmark pathways of inflammatory response, complement, and KRAS signaling up displayed negative interactions with CXCR2. In contrast, CXCR2 was in positive relation to MYC targets v1, base excision repair, proteasome, and DNA replication (Figure $7(\mathrm{~d})$ ). 
TABLE 3: The information of the first 20 KEGG pathways enriched by upregulated CXCR2-relevant genes.

\begin{tabular}{|c|c|c|c|c|c|}
\hline Description & GeneRatio & BgRatio & $p$ value & FDR & Size \\
\hline Staphylococcus aureus infection & $39 / 411$ & $96 / 8009$ & $7.16 E-26$ & $1.90 E-23$ & 39 \\
\hline Hematopoietic cell lineage & $38 / 411$ & $99 / 8009$ & $3.70 E-24$ & $4.90 E-22$ & 38 \\
\hline Phagosome & $44 / 411$ & $152 / 8009$ & $4.61 E-22$ & $3.62 E-20$ & 44 \\
\hline Rheumatoid arthritis & $35 / 411$ & $93 / 8009$ & $5.46 E-22$ & $3.62 E-20$ & 35 \\
\hline Leishmaniasis & $31 / 411$ & $77 / 8009$ & $1.26 E-20$ & $6.68 E-19$ & 31 \\
\hline Tuberculosis & $45 / 411$ & $180 / 8009$ & $1.02 E-19$ & $4.52 E-18$ & 45 \\
\hline Viral protein interaction with cytokine and cytokine receptor & $32 / 411$ & $100 / 8009$ & $9.47 E-18$ & $3.58 E-16$ & 32 \\
\hline Cytokine-cytokine receptor interaction & $54 / 411$ & $294 / 8009$ & $5.80 E-17$ & $1.92 E-15$ & 54 \\
\hline Cell adhesion molecules (CAMs) & $36 / 411$ & $147 / 8009$ & $1.17 E-15$ & $3.46 E-14$ & 36 \\
\hline Osteoclast differentiation & $32 / 411$ & $128 / 8009$ & $2.59 E-14$ & $6.87 E-13$ & 32 \\
\hline Inflammatory bowel disease (IBD) & $23 / 411$ & $65 / 8009$ & $3.66 E-14$ & $8.81 E-13$ & 23 \\
\hline Allograft rejection & $18 / 411$ & $38 / 8009$ & $5.50 E-14$ & $1.22 E-12$ & 18 \\
\hline Chemokine signaling pathway & $38 / 411$ & $189 / 8009$ & $1.71 E-13$ & $3.49 E-12$ & 38 \\
\hline Human $\mathrm{T}$ cell leukemia virus 1 infection & $41 / 411$ & $219 / 8009$ & $2.24 E-13$ & $4.24 E-12$ & 41 \\
\hline Graft-versus-host disease & $18 / 411$ & $41 / 8009$ & $2.87 E-13$ & $5.07 E-12$ & 18 \\
\hline Type I diabetes mellitus & $18 / 411$ & $43 / 8009$ & $7.86 E-13$ & $1.30 E-11$ & 18 \\
\hline Th17 cell differentiation & $27 / 411$ & $107 / 8009$ & $2.29 E-12$ & $3.57 E-11$ & 27 \\
\hline Antigen processing and presentation & $23 / 411$ & $78 / 8009$ & $2.99 E-12$ & $4.40 E-11$ & 23 \\
\hline Influenza A & $34 / 411$ & $170 / 8009$ & $4.03 E-12$ & $5.63 E-11$ & 34 \\
\hline Asthma & $15 / 411$ & $31 / 8009$ & $4.95 E-12$ & $6.57 E-11$ & 15 \\
\hline
\end{tabular}

TABLE 4: The information of the first 20 biological processes enriched by upregulated CXCR2-relevant genes.

\begin{tabular}{|c|c|c|c|c|c|}
\hline Description & GeneRatio & BgRatio & $p$ value & FDR & Size \\
\hline $\mathrm{T}$ cell activation & $98 / 670$ & $483 / 18866$ & $1.24 E-46$ & $4.26 E-43$ & 98 \\
\hline Leukocyte cell-cell adhesion & $86 / 670$ & $364 / 18866$ & $1.85 E-46$ & $4.26 E-43$ & 86 \\
\hline Leukocyte proliferation & $74 / 670$ & $313 / 18866$ & $5.29 E-40$ & $8.13 E-37$ & 74 \\
\hline Neutrophil activation involved in immune response & $89 / 670$ & $490 / 18866$ & $2.89 E-38$ & $3.32 E-35$ & 89 \\
\hline Leukocyte chemotaxis & $63 / 670$ & $232 / 18866$ & $5.31 E-38$ & $4.89 E-35$ & 63 \\
\hline Neutrophil degranulation & $88 / 670$ & $487 / 18866$ & $1.19 E-37$ & $9.12 E-35$ & 88 \\
\hline Cell chemotaxis & $71 / 670$ & $311 / 18866$ & $2.56 E-37$ & $1.68 E-34$ & 71 \\
\hline Regulation of leukocyte proliferation & $63 / 670$ & $240 / 18866$ & $4.88 E-37$ & $2.81 E-34$ & 63 \\
\hline Mononuclear cell proliferation & $68 / 670$ & $286 / 18866$ & $5.87 E-37$ & $3.00 E-34$ & 68 \\
\hline Regulation of leukocyte cell-cell adhesion & $72 / 670$ & $329 / 18866$ & $1.55 E-36$ & $7.13 E-34$ & 72 \\
\hline Positive regulation of cytokine production & $83 / 670$ & $447 / 18866$ & $2.02 E-36$ & $8.45 E-34$ & 83 \\
\hline Lymphocyte proliferation & $67 / 670$ & $283 / 18866$ & $2.69 E-36$ & $1.03 E-33$ & 67 \\
\hline Positive regulation of cell adhesion & $81 / 670$ & $428 / 18866$ & $3.58 E-36$ & $1.27 E-33$ & 81 \\
\hline Myeloid leukocyte migration & $60 / 670$ & $222 / 18866$ & $4.28 E-36$ & $1.41 E-33$ & 60 \\
\hline Response to molecule of bacterial origin & $74 / 670$ & $356 / 18866$ & $6.20 E-36$ & $1.90 E-33$ & 74 \\
\hline Regulation of $\mathrm{T}$ cell activation & $71 / 670$ & $332 / 18866$ & $2.43 E-35$ & $7.00 E-33$ & 71 \\
\hline Regulation of mononuclear cell proliferation & $59 / 670$ & $221 / 18866$ & $3.54 E-35$ & $9.59 E-33$ & 59 \\
\hline Positive regulation of cell activation & $79 / 670$ & $421 / 18866$ & $5.21 E-35$ & $1.33 E-32$ & 79 \\
\hline Regulation of cell-cell adhesion & $80 / 670$ & $439 / 18866$ & $1.67 E-34$ & $4.05 E-32$ & 80 \\
\hline Regulation of lymphocyte proliferation & $58 / 670$ & $219 / 18866$ & $2.19 E-34$ & $5.05 E-32$ & 58 \\
\hline
\end{tabular}

\section{Discussion}

Based on TCGA and GTEx datasets, we observed the abnormal expression of CXCR2 in tumors and paired normal tissues across pancancer. Survival analysis uncovered the prominent prognostic significance of CXCR2 in diverse cancer types. Especially, CXCR2 expression presented marked upregulation in ovarian cancer as well as its upregulation 
TABLE 5: The information of the first 20 KEGG pathways enriched by downregulated CXCR2-relevant genes.

\begin{tabular}{|c|c|c|c|c|c|}
\hline Description & GeneRatio & BgRatio & $p$ value & FDR & Size \\
\hline PPAR signaling pathway & $2 / 9$ & $76 / 8009$ & 0.003064 & 0.085799 & 2 \\
\hline Nitrogen metabolism & $1 / 9$ & $17 / 8009$ & 0.018951 & 0.191907 & 1 \\
\hline Vitamin digestion and absorption & $1 / 9$ & $24 / 8009$ & 0.026662 & 0.191907 & 1 \\
\hline Aldosterone-regulated sodium reabsorption & $1 / 9$ & $37 / 8009$ & 0.040838 & 0.191907 & 1 \\
\hline African trypanosomiasis & $1 / 9$ & $37 / 8009$ & 0.040838 & 0.191907 & 1 \\
\hline Fat digestion and absorption & $1 / 9$ & $41 / 8009$ & 0.045163 & 0.191907 & 1 \\
\hline Neuroactive ligand-receptor interaction & $2 / 9$ & $340 / 8009$ & 0.053093 & 0.191907 & 2 \\
\hline Cholesterol metabolism & $1 / 9$ & $50 / 8009$ & 0.054831 & 0.191907 & 1 \\
\hline Melanoma & $1 / 9$ & $72 / 8009$ & 0.078097 & 0.24297 & 1 \\
\hline Protein digestion and absorption & $1 / 9$ & $95 / 8009$ & 0.101876 & 0.251426 & 1 \\
\hline Pancreatic secretion & $1 / 9$ & $102 / 8009$ & 0.109004 & 0.251426 & 1 \\
\hline Leukocyte transendothelial migration & $1 / 9$ & $112 / 8009$ & 0.119099 & 0.251426 & 1 \\
\hline Systemic lupus erythematosus & $1 / 9$ & $133 / 8009$ & 0.13997 & 0.251426 & 1 \\
\hline Cell adhesion molecules (CAMs) & $1 / 9$ & $147 / 8009$ & 0.153638 & 0.251426 & 1 \\
\hline Breast cancer & $1 / 9$ & $147 / 8009$ & 0.153638 & 0.251426 & 1 \\
\hline Gastric cancer & $1 / 9$ & $149 / 8009$ & 0.155575 & 0.251426 & 1 \\
\hline Hepatitis C & $1 / 9$ & $155 / 8009$ & 0.161361 & 0.251426 & 1 \\
\hline Tight junction & $1 / 9$ & $169 / 8009$ & 0.174727 & 0.251426 & 1 \\
\hline Influenza A & $1 / 9$ & $170 / 8009$ & 0.175674 & 0.251426 & 1 \\
\hline Alcoholism & $1 / 9$ & $184 / 8009$ & 0.188836 & 0.251426 & 1 \\
\hline
\end{tabular}

TABLE 6: The information of the first 20 biological processes enriched by downregulated CXCR2-relevant genes.

\begin{tabular}{|c|c|c|c|c|c|}
\hline Description & GeneRatio & BgRatio & $p$ value & FDR & Size \\
\hline Forebrain regionalization & $2 / 18$ & $24 / 18866$ & 0.000234 & 0.105833 & 2 \\
\hline Triglyceride catabolic process & $2 / 18$ & $38 / 18866$ & 0.000592 & 0.105833 & 2 \\
\hline Regulation of gastrulation & $2 / 18$ & $43 / 18866$ & 0.000759 & 0.105833 & 2 \\
\hline Neutral lipid catabolic process & $2 / 18$ & $48 / 18866$ & 0.000945 & 0.105833 & 2 \\
\hline Acylglycerol catabolic process & $2 / 18$ & $48 / 18866$ & 0.000945 & 0.105833 & 2 \\
\hline Regulation of sodium ion transmembrane transporter activity & $2 / 18$ & $55 / 18866$ & 0.001239 & 0.110357 & 2 \\
\hline Regulation of sodium ion transmembrane transport & $2 / 18$ & $65 / 18866$ & 0.001726 & 0.110357 & 2 \\
\hline Neural retina development & $2 / 18$ & $72 / 18866$ & 0.002112 & 0.110357 & 2 \\
\hline Regulation of cardiac conduction & $2 / 18$ & $73 / 18866$ & 0.002171 & 0.110357 & 2 \\
\hline Glycerolipid catabolic process & $2 / 18$ & $74 / 18866$ & 0.00223 & 0.110357 & 2 \\
\hline Regulation of sodium ion transport & $2 / 18$ & $88 / 18866$ & 0.003135 & 0.110357 & 2 \\
\hline Axis specification & $2 / 18$ & $88 / 18866$ & 0.003135 & 0.110357 & 2 \\
\hline Regulation of neural precursor cell proliferation & $2 / 18$ & $91 / 18866$ & 0.003348 & 0.110357 & 2 \\
\hline Dorsal/ventral pattern formation & $2 / 18$ & $92 / 18866$ & 0.003421 & 0.110357 & 2 \\
\hline Triglyceride metabolic process & $2 / 18$ & $110 / 18866$ & 0.004849 & 0.110357 & 2 \\
\hline Endocrine system development & $2 / 18$ & $125 / 18866$ & 0.006216 & 0.110357 & 2 \\
\hline Camera-type eye morphogenesis & $2 / 18$ & $125 / 18866$ & 0.006216 & 0.110357 & 2 \\
\hline Regulation of embryonic development & $2 / 18$ & $134 / 18866$ & 0.007111 & 0.110357 & 2 \\
\hline Neutral lipid metabolic process & $2 / 18$ & $138 / 18866$ & 0.007527 & 0.110357 & 2 \\
\hline Acylglycerol metabolic process & $2 / 18$ & $138 / 18866$ & 0.007527 & 0.110357 & 2 \\
\hline
\end{tabular}

contributed to more undesirable survival outcomes. Hence, CXCR2 might act as a promising prognostic predictor of ovarian cancer. The response of ovarian cancer to immunotherapeutic agents remains limited. Although immunother- apy may produce a long-lasting response in a few patients, most of the patients do not respond to this therapy, covering those with PD-L1-expressed tumors [37]. Nevertheless, evaluating the sensitivity or resistance to target therapeutic 


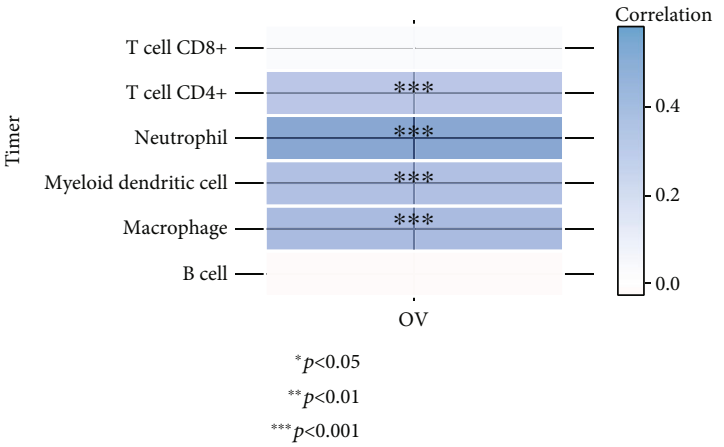

(a)

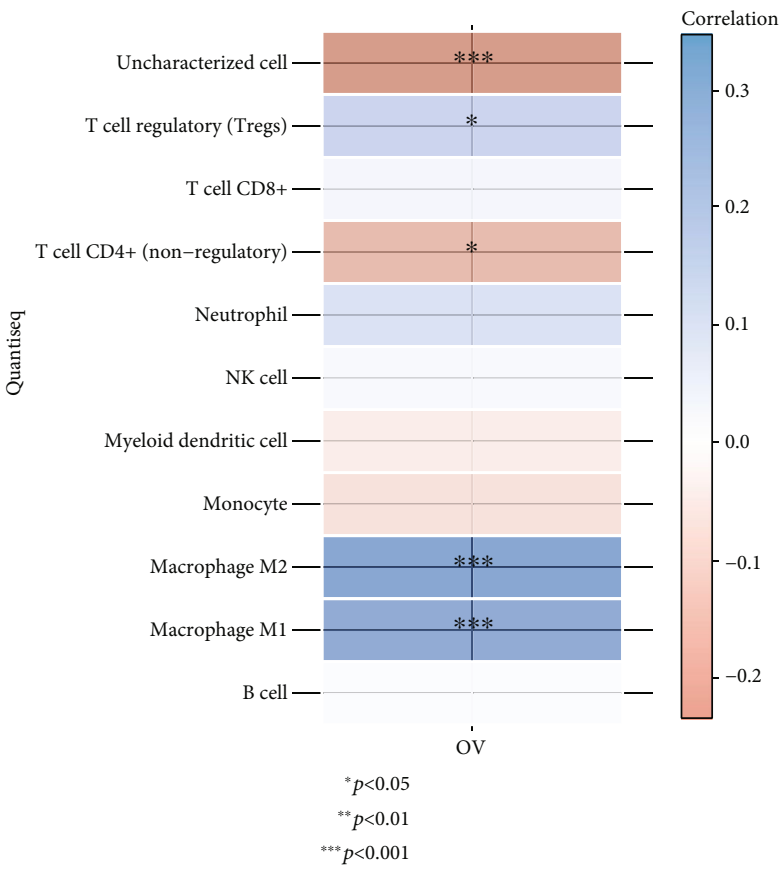

(b)

Figure 6: Continued. 


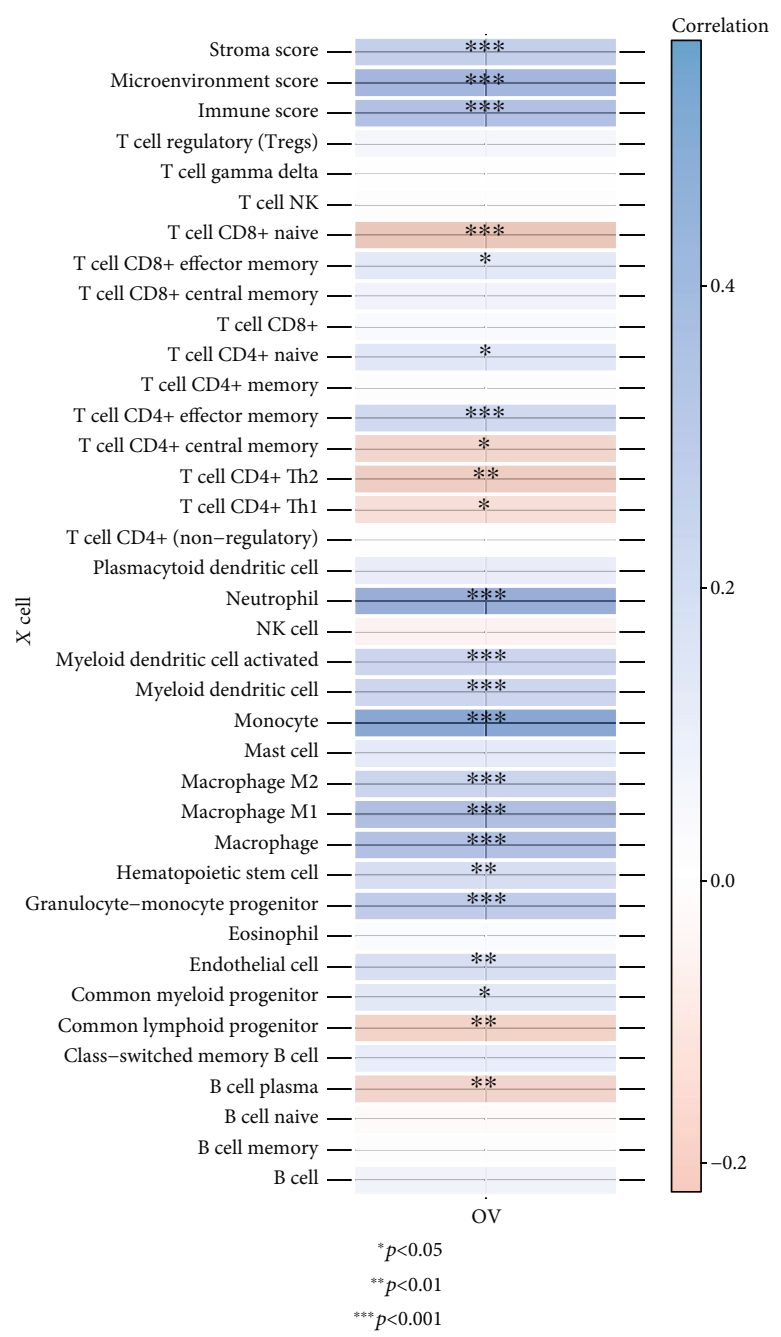

(c)

FIGURE 6: Analysis of interactions between CXCR2 and immune cell infiltration in tumor microenvironment. (a) Correlations of CXCR2 with the abundance of immune cells in ovarian cancer through TIMER algorithm. (b) Associations of CXCR2 with the abundance of immune cells across ovarian cancer tissues with quanTIseq algorithm. (c) Associations between CXCR2 and infiltration levels of immune cells in ovarian cancer utilizing xCell algorithm. Red meant positive correlation while blue meant negative correlation. ${ }^{*} p<0.05 ;{ }^{* *} p<$ $0.01 ;{ }^{* * *} p<0.001$.

populations according to stratification by cancer markers including TMB, PD-L1, tumor-infiltrating lymphocytes, and neoantigens can enhance the predictive efficacy of immunotherapeutic response [38]. Our pancancer analysis demonstrated the close interactions of CXCR2 with immune checkpoints, neoantigen, TMB, and MSI, indicating that CXCR2 could participate in modulating immune response. Our genetic mutation analysis uncovered that there occurred widespread mutations of CXCR2 across pancancer. Amplification was the major mutational type of CXCR2 in ovarian cancer. This indicated that CXCR2 amplification contributed to its overexpression in ovarian cancer.

Under the cutoffs of $\mid \log 2$ fold change $\mid>1$ and FDR $<$ 0.05, we identified 734 CXCR2-relevant genes. We noticed that CXCR2-relevant genes were markedly enriched in immunity activation such as Th17 cell differentiation, cytokine-cytokine receptor interaction, chemokine signaling pathway, and antigen processing and presentation as well as carcinogenic pathways such as PPAR signaling pathway. For instance, PARP inhibitor has emerged as a therapeutic agent against ovarian cancer according to the DNA repair vulnerability in ovarian cancer cells, which prevents the repair of DNA single-strand break as well as has generated doublestrand break that is unable to be precisely repaired in tumor cells [39].

Based on three algorithms (TIMER, quanTIseq, and $\mathrm{xCell}$ ), we noticed the prominent interaction between CXCR2 and macrophage in ovarian cancer tissues. Macrophage constitutes a key component of the tumor microenvironment [13]. Tumor-associated macrophage is macrophage produced by the infiltrations of peripheral blood mononuclear cells into solid tumor tissues, occupying a large part of tumor stromal cells [13]. Because of the increased plasticity and heterogeneity of macrophage, it has distinct biological 


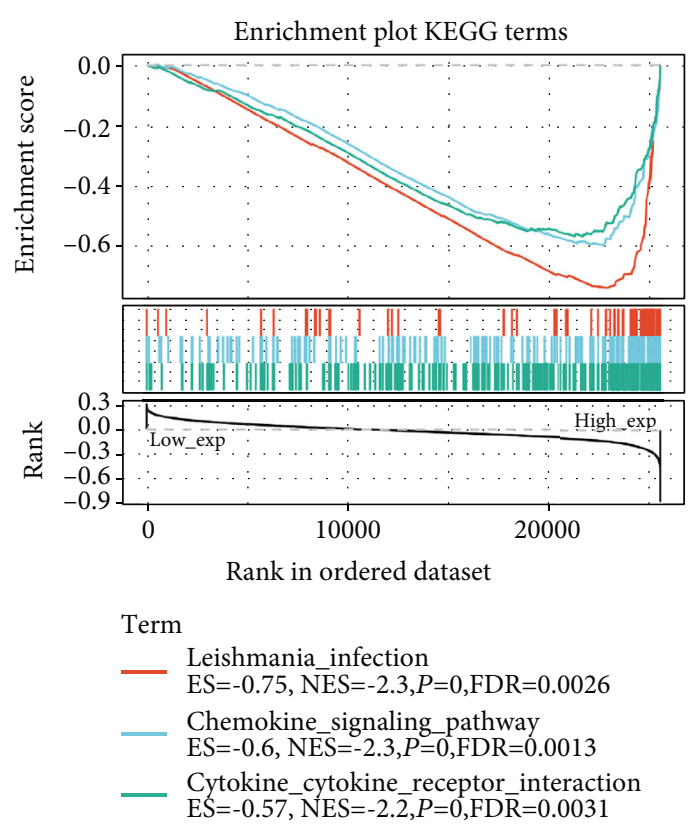

(a)

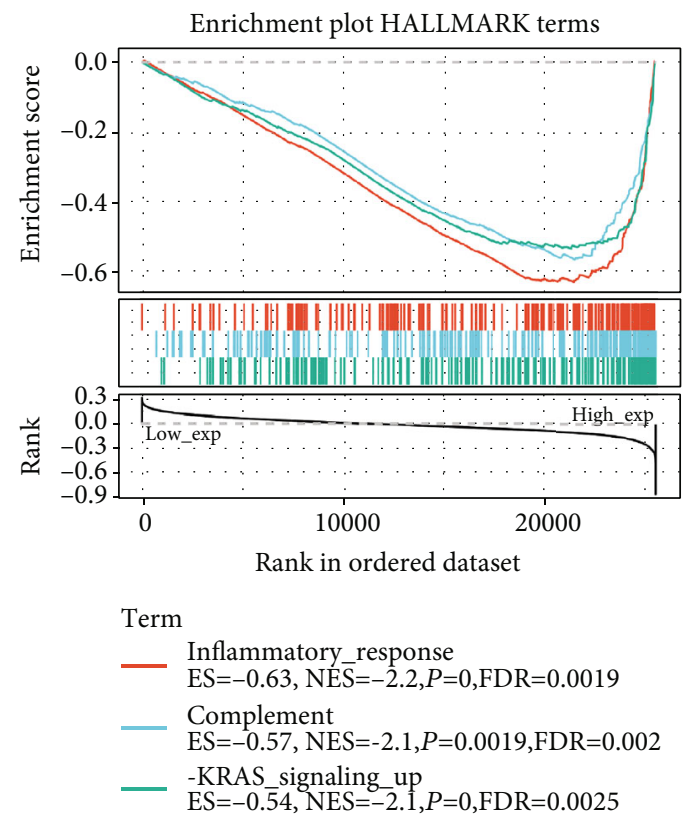

(c)

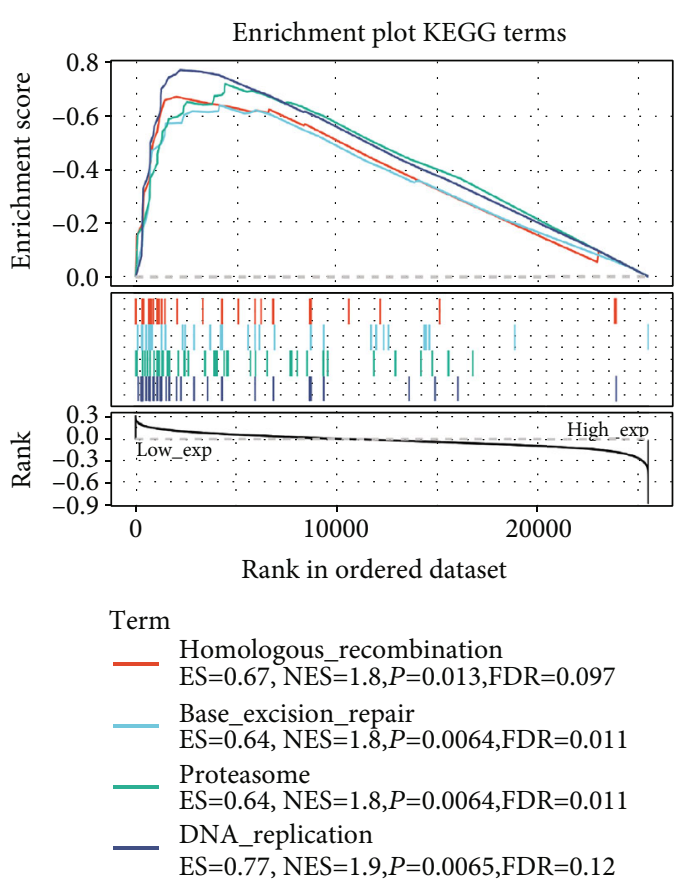

(b)

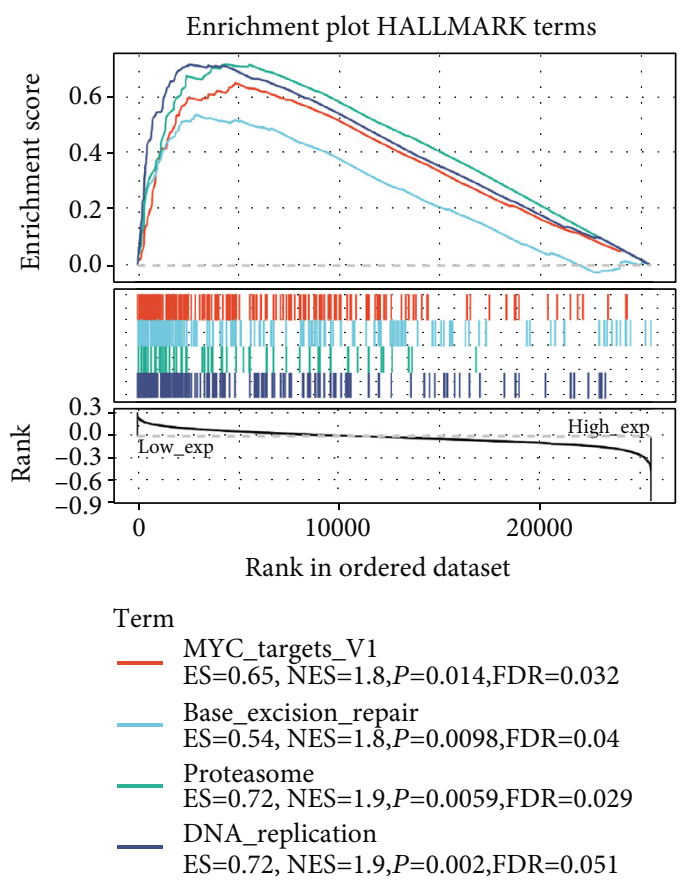

(d)

FIGURE 7: GSEA of CXCR2 linked with KEGG and hallmark pathways. (a) GSEA results of CXCR2 ranked in the first three for its negative associations with KEGG pathways. (b) GSEA results of CXCR2 ranked in the first four for its positive associations with KEGG pathways. (c) GSEA results of CXCR2 ranked in the first three for its negative associations with hallmark pathways. (d) GSEA results of CXCR2 ranked in the first four or its positive associations with hallmark pathways. ES: enrichment score; NES: nominal enrichment score; FDR: false discovery rate.

functions in diverse tumor microenvironment, including two major phenotypes: M1 and M2 macrophages [40]. Tumorassociated macrophage is abundant in the ovarian cancer microenvironment and affects patients' survival outcomes [40]. The relationship of CXCR2 with macrophage has been reported in several cancer types. For instance, macrophage reeducation by CXCR2 inhibitors may drive senescence as well as suppress tumor progression in advanced prostate cancer [41]. CXCR2-dominated interplays between cancer cells and macrophages drive gastric cancer metastases [42]. 
CXCR2 was mainly involved in modulating chemokine signaling pathway, cytokine-cytokine receptor interaction, inflammatory response, and complement as well as DNA damage repair. CXCR2 produced by cancer cells induce neutrophil extracellular traps, which interferes with immune cytotoxicity [43]. CXCR2-modified CAR-T cells enhance trafficking capacity, which improves therapeutic response in hepatocellular carcinoma [44]. Blockage of CXCR2 may enhance the sensitivity and effectiveness of immunotherapy and suppress tumor progression [18]. Combining previous evidence, CXCR2 may exert a critical role in modulating immune response. Nevertheless, there are several limitations in our study. The regulatory functions of CXCR2 in ovarian carcinogenesis and tumor immunity will be investigated in in vitro and in vivo experiments. Moreover, prognostic significance of CXCR2 expression should be verified in a larger ovarian cancer cohort.

\section{Conclusion}

Collectively, our integrative analysis of CXCR2 uncovered the prominent associations of CXCR2 expression with survival outcomes, immune cell infiltration, and immune response in ovarian cancer, which could contribute to explain the function of CXCR2 in carcinogenesis and immunotherapeutic response from various perspectives.

\author{
Abbreviations \\ TMB: $\quad$ Tumor mutation burden \\ CXCR: Chemokine receptor \\ TCGA: The Cancer Genome Atlas \\ GDC: Genomic Data Commons \\ GTEx: Genotype-Tissue Expression \\ GEPIA2: Gene Expression Profiling Interactive Analysis \\ 2 \\ OS: $\quad$ Overall survival \\ DSS: Disease-specific survival \\ MSI: $\quad$ Microsatellite instability \\ cBioPortal: cBio Cancer Genomics Portal \\ FDR: $\quad$ False discovery rate \\ GO: $\quad$ Gene Ontology \\ KEGG: $\quad$ Kyoto Encyclopedia of Genes and Genomes \\ TIMER: Tumor Immune Estimation Resource \\ GSEA: $\quad$ Gene set enrichment analysis \\ MsigDB: Molecular Signature Database \\ NES: $\quad$ Nominal enrichment score.
}

\section{Data Availability}

The data used to support the findings of this study are included within the supplementary information files.

\section{Conflicts of Interest}

The authors declare no conflicts of interest.

\section{Authors' Contributions}

Haizhou Ji and Mi Ren contributed equally to this work.

\section{Acknowledgments}

This work was supported by the Innovation Project of the Department of Health of Fujian Province (2017-CX-9).

\section{Supplementary Materials}

Supplementary Figure 1: prognostic implication of CXCR2 in diverse cancer types. (A) Kaplan-Meier curves of the OS differences between high and low expression of CXCR2 groups for ACC, DLBC, LAML, LGG, OV, and MESO. (B) Kaplan-Meier curves of the DSS differences between high and low expression of CXCR2 groups for ACC, LGG, OV, and STAD. (Supplementary Materials)

\section{References}

[1] S. Zhang, B. Xie, L. Wang et al., "Macrophage-mediated vascular permeability via VLA4/VCAM1 pathway dictates ascites development in ovarian cancer," The Journal of Clinical Investigation, vol. 131, no. 3, 2021.

[2] Y. Wang, G. Zhao, S. Condello et al., "Frizzled-7 identifies platinum-tolerant ovarian cancer cells susceptible to ferroptosis," Cancer Research, vol. 81, no. 2, pp. 384-399, 2021.

[3] L. Hao, J. M. Wang, B. Q. Liu et al., "m6A-YTHDF1-mediated TRIM29 upregulation facilitates the stem cell-like phenotype of cisplatin-resistant ovarian cancer cells," Biochimica et Biophysica Acta (BBA) - Molecular Cell Research, vol. 1868, no. 1 , article $118878,2021$.

[4] Q. Lin, W. Liu, S. Xu et al., "PARP inhibitors as maintenance therapy in newly diagnosed advanced ovarian cancer: a meta-analysis," BJOG, vol. 128, no. 3, pp. 485-493, 2021.

[5] F. A. Raja, N. Chopra, and J. A. Ledermann, "Optimal first-line treatment in ovarian cancer," Annals of Oncology, vol. 23, Suppl 10, pp. x118-x127, 2012.

[6] V. Gogineni, S. Morand, H. Staats et al., "Current ovarian cancer maintenance strategies and promising new developments," Journal of Cancer, vol. 12, no. 1, pp. 38-53, 2021.

[7] M. Zhang, S. Cheng, Y. Jin, Y. Zhao, and Y. Wang, "Roles of CA125 in diagnosis, prediction, and oncogenesis of ovarian cancer," Biochimica Et Biophysica Acta. Reviews on Cancer, vol. 1875, no. 2, article 188503, 2021.

[8] A. Mukherjee, C. Y. Chiang, H. A. Daifotis et al., "Adipocyteinduced FABP4 expression in ovarian cancer cells promotes metastasis and mediates carboplatin resistance," Cancer Research, vol. 80, no. 8, pp. 1748-1761, 2020.

[9] Y. Jiang, C. Wang, and S. Zhou, "Targeting tumor microenvironment in ovarian cancer: premise and promise," Biochimica Et Biophysica Acta. Reviews on Cancer, vol. 1873, no. 2, article 188361, 2020.

[10] J. Gong, A. Chehrazi-Raffle, S. Reddi, and R. Salgia, "Development of PD-1 and PD-L1 inhibitors as a form of cancer immunotherapy: a comprehensive review of registration trials and future considerations," Journal for Immunotherapy of Cancer, vol. 6 , no. 1, p. 8, 2018.

[11] L. Xia, Y. Wang, S. Cai, and M. Xu, "DGAT1 expression promotes ovarian cancer progression and is associated with poor prognosis," Journal of Immunology Research, vol. 2021, Article ID 6636791, 10 pages, 2021. 
[12] Y. T. Liu and Z. J. Sun, "Turning cold tumors into hot tumors by improving T-cell infiltration,” Theranostics, vol. 11, no. 11, pp. 5365-5386, 2021.

[13] Y. An and Q. Yang, "Tumor-associated macrophage-targeted therapeutics in ovarian cancer," International Journal of Cancer, vol. 149, no. 1, pp. 21-30, 2021.

[14] Y. L. Zhang, H. J. Cao, X. Han et al., "Chemokine receptor CXCR-2 initiates atrial fibrillation by triggering monocyte mobilization in mice," Hypertension, vol. 76, no. 2, pp. 381392, 2020.

[15] Y. Cheng, X. L. Ma, Y. Q. Wei, and X. W. Wei, "Potential roles and targeted therapy of the CXCLs/CXCR2 axis in cancer and inflammatory diseases," Biochimica Et Biophysica Acta. Reviews on Cancer, vol. 1871, no. 2, pp. 289-312, 2019.

[16] T. M. Nywening, B. A. Belt, D. R. Cullinan et al., "Targeting both tumour-associated CXCR2(+) neutrophils and CCR2(+) macrophages disrupts myeloid recruitment and improves chemotherapeutic responses in pancreatic ductal adenocarcinoma," Gut, vol. 67, no. 6, pp. 1112-1123, 2018.

[17] J. Yang, C. Yan, A. E. Vilgelm et al., "Targeted deletion of CXCR2 in myeloid cells alters the tumor immune environment to improve antitumor immunity," Cancer Immunology Research, vol. 9, no. 2, pp. 200-213, 2021.

[18] W. Zhang, H. Wang, M. Sun et al., "CXCL5/CXCR2 axis in tumor microenvironment as potential diagnostic biomarker and therapeutic target," Cancer Communications, vol. 40, no. 2-3, pp. 69-80, 2020.

[19] G. Yang, D. G. Rosen, G. Liu et al., "CXCR2 promotes ovarian cancer growth through dysregulated cell cycle, diminished apoptosis, and enhanced angiogenesis," Clinical Cancer Research, vol. 16, no. 15, pp. 3875-3886, 2010.

[20] Y. L. Dong, S. M. Kabir, E. S. Lee, and D. S. Son, "CXCR2driven ovarian cancer progression involves upregulation of proinflammatory chemokines by potentiating NF- $\kappa$ B activation via EGFR-transactivated Akt signaling," PLoS One, vol. 8, no. 12, article e83789, 2013.

[21] T. B. Henriques, D. Z. dos Santos, I. dos Santos Guimarães et al., "Inhibition of CXCR2 plays a pivotal role in resensitizing ovarian cancer to cisplatin treatment," Aging (Albany NY), vol. 13, no. 10, pp. 13405-13420, 2021.

[22] M. A. Jensen, V. Ferretti, R. L. Grossman, and L. M. Staudt, "The NCI Genomic Data Commons as an engine for precision medicine," Blood, vol. 130, no. 4, pp. 453-459, 2017.

[23] Z. Tang, B. Kang, C. Li, T. Chen, and Z. Zhang, "GEPIA2: an enhanced web server for large-scale expression profiling and interactive analysis," Nucleic Acids Research, vol. 47, no. W1, pp. W556-w560, 2019.

[24] S. L. Topalian, J. M. Taube, R. A. Anders, and D. M. Pardoll, "Mechanism-driven biomarkers to guide immune checkpoint blockade in cancer therapy," Nature Reviews. Cancer, vol. 16, no. 5, pp. 275-287, 2016.

[25] Z. R. Chalmers, C. F. Connelly, D. Fabrizio et al., "Analysis of 100,000 human cancer genomes reveals the landscape of tumor mutational burden," Genome Medicine, vol. 9, no. 1, p. 34, 2017.

[26] F. Chen, Z. Zou, J. du et al., "Neoantigen identification strategies enable personalized immunotherapy in refractory solid tumors," The Journal of Clinical Investigation, vol. 129, no. 5, pp. 2056-2070, 2019.

[27] R. J. Hause, C. C. Pritchard, J. Shendure, and S. J. Salipante, "Classification and characterization of microsatellite instabil- ity across 18 cancer types," Nature Medicine, vol. 22, no. 11, pp. 1342-1350, 2016.

[28] A. Mayakonda, D. C. Lin, Y. Assenov, C. Plass, and H. P. Koeffler, "Maftools: efficient and comprehensive analysis of somatic variants in cancer," Genome Research, vol. 28, no. 11, pp. 1747-1756, 2018.

[29] E. Cerami, J. Gao, U. Dogrusoz et al., "The cBio cancer genomics portal: an open platform for exploring multidimensional cancer genomics data," Cancer Discovery, vol. 2, no. 5, pp. 401404, 2012.

[30] M. E. Ritchie, B. Phipson, D. Wu et al., "limma powers differential expression analyses for RNA-sequencing and microarray studies," Nucleic Acids Research, vol. 43, no. 7, article e47, 2015.

[31] G. Yu, L. G. Wang, Y. Han, and Q. Y. He, “clusterProfiler: an R package for comparing biological themes among gene clusters," OMICS, vol. 16, no. 5, pp. 284-287, 2012.

[32] T. Li, J. Fan, B. Wang et al., "TIMER: a web server for comprehensive analysis of tumor-infiltrating immune cells," Cancer Research, vol. 77, no. 21, pp. e108-e110, 2017.

[33] F. Finotello, C. Mayer, C. Plattner et al., "Molecular and pharmacological modulators of the tumor immune contexture revealed by deconvolution of RNA-seq data," Genome Medicine, vol. 11, no. 1, p. 34, 2019.

[34] D. Aran, Z. Hu, and A. J. Butte, "xCell: digitally portraying the tissue cellular heterogeneity landscape," Genome Biology, vol. 18, no. 1, p. 220, 2017.

[35] A. Subramanian, P. Tamayo, V. K. Mootha et al., "Gene set enrichment analysis: a knowledge-based approach for interpreting genome-wide expression profiles," Proceedings of the National Academy of Sciences of the United States of America, vol. 102, no. 43, pp. 15545-15550, 2005.

[36] A. Liberzon, A. Subramanian, R. Pinchback, H. Thorvaldsdottir, P. Tamayo, and J. P. Mesirov, "Molecular signatures database (MSigDB) 3.0," Bioinformatics, vol. 27, no. 12, pp. 1739-1740, 2011.

[37] X. Li, L. Luo, M. Jiang et al., “Cocktail strategy for 'cold' tumors therapy via active recruitment of CD8+ T cells and enhancing their function," Journal of Controlled Release, vol. 334, pp. 413-426, 2021.

[38] S. Morand, M. Devanaboyina, H. Staats, L. Stanbery, and J. Nemunaitis, "Ovarian cancer immunotherapy and personalized medicine," International Journal of Molecular Sciences, vol. 22, no. 12, p. 6532, 2021.

[39] R. E. Miller, A. Leary, C. L. Scott et al., "ESMO recommendations on predictive biomarker testing for homologous recombination deficiency and PARP inhibitor benefit in ovarian cancer," Annals of Oncology, vol. 31, no. 12, pp. 1606-1622, 2020.

[40] H. Xia, S. Li, X. Li et al., "Autophagic adaptation to oxidative stress alters peritoneal residential macrophage survival and ovarian cancer metastasis," JCI Insight, vol. 5, no. 18, 2020.

[41] D. di Mitri, M. Mirenda, J. Vasilevska et al., "Re-education of tumor-associated macrophages by CXCR2 blockade drives senescence and tumor inhibition in advanced prostate cancer," Cell Reports, vol. 28, no. 8, pp. 2156-2168.e5, 2019.

[42] Z. Zhou, G. Xia, Z. Xiang et al., "A C-X-C chemokine receptor type 2-dominated cross-talk between tumor cells and macrophages drives gastric cancer metastasis," Clinical Cancer Research, vol. 25, no. 11, pp. 3317-3328, 2019. 
[43] Á. Teijeira, S. Garasa, M. Gato et al., "CXCR1 and CXCR2 chemokine receptor agonists produced by tumors induce neutrophil extracellular traps that interfere with immune cytotoxicity," Immunity, vol. 52, no. 5, pp. 856-871.e8, 2020.

[44] G. Liu, W. Rui, H. Zheng et al., "CXCR2-modified CAR-T cells have enhanced trafficking ability that improves treatment of hepatocellular carcinoma," European Journal of Immunology, vol. 50, no. 5, pp. 712-724, 2020. 